\title{
Jump Discontinuities of Semilinear, Strictly Hyperbolic Systems in Two Variables: Creation and Propagation
}

\author{
Jeffrey Rauch ${ }^{1 \star}$ and Michael Reed ${ }^{2 \star \star}$
}

1 Department of Mathematics, University of Michigan, Ann Arbor, MI 48109, USA

2 Department of Mathematics, Duke University, Durham, NC 27706, USA

\begin{abstract}
The creation and propagation of jump discontinuities in the solutions of semilinear strictly hyperbolic systems is studied in the case where the initial data has a discrete set, $\left\{x_{i}\right\}_{i=1}^{n}$, of jump discontinuities. Let $S$ be the smallest closed set which satisfies:

(i) $S$ is a union of forward characteristics.

(ii) $S$ contains all the forward characteristics from the points $\left\{x_{i}\right\}_{i=1}^{n}$.

(iii) if two forward characteristics in $S$ intersect, then all forward characteristics from the point of intersection lie in $S$.

We prove that the singular support of the solution lies in $S$. We derive a sum law which gives a lower bound on the smoothness of the solution across forward characteristics from an intersection point. We prove a sufficient condition which guarantees that in many cases the lower bound is also an upper bound.
\end{abstract}

\section{Introduction}

This paper is devoted to the study of the regularity of locally bounded solutions to strictly hyperbolic semilinear first order systems in one space variable. That is, we study $u \in L_{\mathrm{loc}}^{\infty}(\Omega)$ satisfying

$$
A_{0}(x, t) \partial_{t} u-A_{1}(x, t) \partial_{x} u=G(x, t, u)
$$

where the $A_{i}$ are smooth $m \times m$ complex matrix-valued functions. We suppose that the system is strictly hyperbolic, that is, $\operatorname{det} A_{0} \neq 0$ and the equation $\operatorname{det}\left(A_{0}-\lambda A_{1}\right)=0$ has $m$ distinct real roots, $\left\{\lambda_{i}\right\}_{i=1}^{m}$, for all $\langle x, t\rangle$ under consideration. We study solutions on $R_{T}$, the open trapezodial region bounded above and below by the lines $t=T, t=0$, on the left by a characteristic of maximal speed, and on the right by a characteristic of minimal speed. We let $I_{i}=R_{T} \cap\{\langle x, t\rangle \mid t=\bar{t}\}$.

If $u \in L^{\infty}\left(R_{T}\right)$ satisfies (1.1) in the sense of distributions, then $u$ is weakly continuous on $[0, T]$ with values in $L^{\infty}$ in the sense that for any fixed $\tilde{t} \in(0, T)$ and

* Research partially supported by NSF Grant \# MCS-79-01857

** Research partially supported by NSF Grant \# MCS $-78-02179$ 


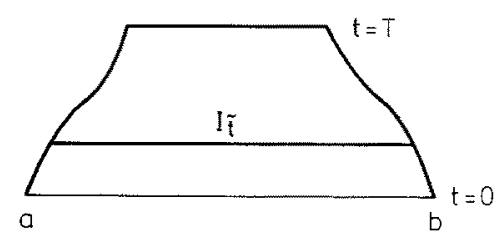

Fig. 1. The region $R_{T}$

$\phi \in \mathscr{D}\left(\right.$ Interior $\left.\left(I_{t}\right)\right), \phi u$ is a continuous function of $t$, for $t$ near $\tilde{t}$, with values in $L^{\infty}(R)=L^{1}(R)^{*}$ endowed with the weak star topology. Similar one sided assertions apply at $t=T$ and $t=0$ so it makes sense to discuss the initial values of $u$. In addition there is a local existence and uniqueness theorem in this category, and for many systems solutions exist globally in time. Thus, there are an abundance of $L^{\infty}$ solutions of (1.1) and they are uniquely determined on $R_{T}$ by their data on $I_{0}=[a, b]$.

In our analysis we take advantage of the fact that by a smooth change of the dependent variables, the Eq. (1.1) and the initial condition can be cast in the canonical form:

$$
\begin{aligned}
\left(\partial_{t}+\Lambda \partial_{x}\right) u & =F(x, t, u) \quad\langle x, t\rangle \in R_{T} \\
u(x, 0) & =u^{0}(x) \quad x \in I_{0}
\end{aligned}
$$

where $A=\operatorname{diag}\left\{\lambda_{1}, \ldots, \lambda_{m}\right\}$.

Now, suppose that the initial data $u^{0}$ is $C^{\infty}$ with bounded derivatives except for finitely many jump discontinuities (either in $u$ or derivatives of $u$ or both) at the points $x_{1}, \ldots, x_{n} \in I_{0}$. What are the smoothness properties of $u$ in $R_{T}$ ? Let $S_{0}$ denote the intersection of $R_{T}$ with the union of forward characteristic curves from each of the points $x_{i}$. If $F$ is linear function of $u$ for each $\langle x, t\rangle$, then the singular support of $u$ in $R$ will be contained in $S_{0}$. In [4], we gave the following example which showed that if $F$ is nonlinear the singular support may be larger than $S_{0}$. Consider the system

$$
\begin{array}{rlrl}
\left(\frac{\partial}{\partial t}+\frac{\partial}{\partial x}\right) v & =0, & v(x, 0) & =\chi_{\{x \mid x \leqq-1\}} \\
\left(\frac{\partial}{\partial t}-\frac{\partial}{\partial x}\right) w & =0, & w(x, 0) & =\chi_{\{x \mid x \geqq 1\}} \\
\left(\frac{\partial}{\partial t}\right) z & =v w, & z(x, 0) \equiv 0 .
\end{array}
$$

Then it is easy to check directly that $u=\langle v, w, z\rangle$ jumps across the solid lines in Fig. 2. Furthermore, although $u$ is continuous across the dashed line, $D_{x} u$ has a jump there. This new "anomalous" discontinuity across the dashed line could not occur if the right-hand of (1.3) were linear. This suggests the following general principle for semilinear hyperbolic partial differential equations: When two singularity bearing characteristics cross, the point of intersection becomes, 


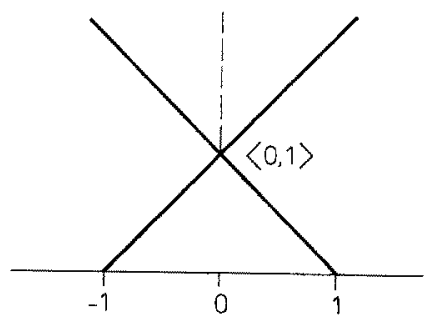

Fig. 2

in general, a source of new singularities traveling on all forward characteristics from that point. Notice that in the example (1.3), the anomalous singularity is in the first derivative while the singularities along the solid lines are in $u$ itself. This suggests a second general principle: Anomalous singularities are weaker than the singularities that produced them. It is the purpose of this paper to make these two principles precise for jump discontinuities of solutions to semilinear strictly hyperbolic systems in two variables.

From the intersection points of $S_{0}$, construct the forward characteristics which are not already in $S_{0}$ and call this set $S_{1}$. In general, let $S_{n+1}$ denote the union of forward characteristics, which are not already in $\bigcup_{k=0}^{n} S_{k}$, from intersection points of $\bigcup_{k=0}^{n} S_{k}$. Define $S$ to be the closure of $\bigcup^{\infty} S_{k}$ intersected with $R_{T}$. Examples of such sets $S_{i}$ are depicted in Figs. 3 and 4. Figure 3 shows the set $S$ for the system (1.3) with two initial singular points $x_{1}$ and $x_{2}$. In general, $\bigcup S_{k}$ will not be a closed set. This can happen as follows. Consider a four-by-four system with two rightward moving and two leftward moving characteristics and two initial singular points $x_{1}$ and $x_{2}$. The point $p$ (See Fig. 4) will be a limit point of intersection points and it is quite easy to see that whenever that is the case, the curves of $\bigcup^{\infty} S_{k}$ will be dense in the whole forward cone from $p$ (i.e. everything to the right of the most

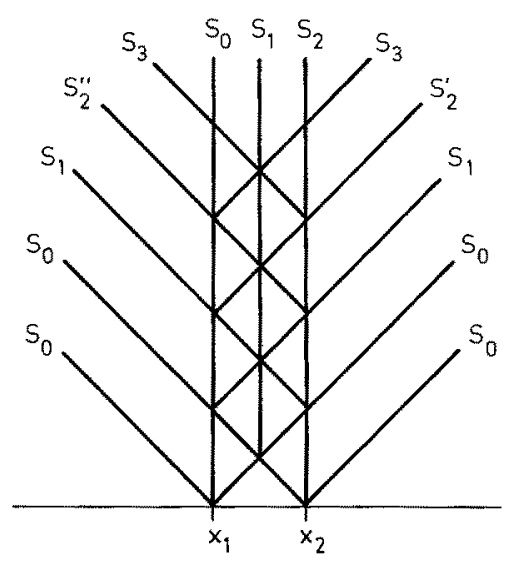

Fig. 3

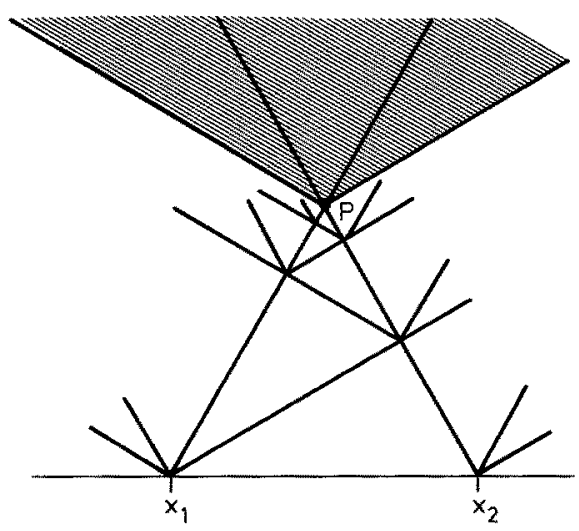

Fig. 4 


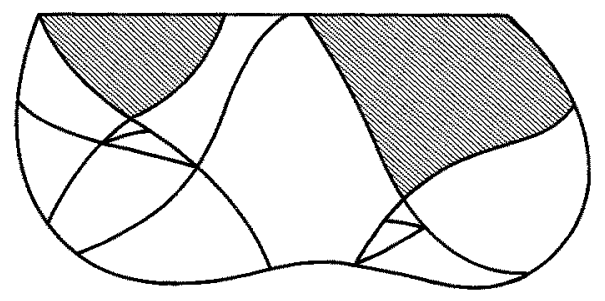

Fig. 5. Part of a typical set $S$

leftward characteristic and to the left of the most rightward characteristic), so the whole forward cone from $p$ will be in $S$. In general, $S$ will consist of such closed forward cones plus a network of pieces of characteristic curves (see Fig. 5). There is a qualitative difference between the cases $m=2, m=3$, and $m \geqq 4$. In the case $m=2$, singularities propagate as in the linear case (this is just the result of [4]) since $S=S_{0}$. In the case $m=3$, anomalous singularities can occur but $S$ will consist of a locally finite latticework of curves. For $m \geqq 4, S$ will contain, in general, both a latticework of curves and closed forward cones.

Now we can describe the results of this paper. In Sect. 2, we prove that $u$ is $C^{\infty}$ on $R_{T} \backslash S$, i.e. the singular support of $u$ is contained in $S$. Thus, the only way that anomalous singularities (that is, singularities not present if $F$ is linear) can appear is by the method described in the first general principle above.

In Sect. 3, we investigate the second general principle, the amelioration of anomalous singularities. We will say that $u$ has order $n$ across $a$ curve in $S$ if all of the first $n$ partial derivatives of $u$ are continuous across the curve but the same is not true for $n+1$. Suppose that two characteristics $\mathscr{C}_{1}$ and $\mathscr{C}_{2}$ carrying singularities of order $n_{1}$ and $n_{2}$ respectively intersect at a point $p$ and that $u$ is smooth across the other characteristics which are incoming to $p$. We prove that $u$ will be at least of order $n_{1}+n_{2}+2$ on all of the forward characteristics from $p$ except $\mathscr{C}_{1}$ and $\mathscr{C}_{2}$ (where $u$ will still have orders $n_{1}$ and $n_{2}$ ). In general, more than two singularity bearing characteristics may intersect at a single point. Label the characteristics coming in to $p$ by $\mathscr{C}_{i}, i=1, \ldots, m$, and the orders of $u$ across the incoming parts of the $\mathscr{C}_{i}$ by $n_{i}, i=1, \ldots, m$, where we have chosen our labels so that $n_{1} \leqq n_{2} \leqq n_{i}$, for $i \neq 1,2$. If $u$ is $C^{\infty}$ across $\mathscr{C}_{i}$ then we set $n_{i}=\infty$. Denote by $m_{i}$ the order of $u$ across the outgoing piece of $\mathscr{C}_{i}$. We prove that

$$
m_{i} \geqq \min \left\{n_{i}, n_{1}+n_{2}+2\right\} .
$$

In other words, if $n_{1}$ and $n_{2}$ are the orders of the worst incoming singularities then the outgoing singularities will have order at least $n_{1}+n_{2}+2$ unless $u$ has order an $n_{i}$ which is less than $n_{1}+n_{2}+2$ on the incoming part of the characteristic in question. In the example (1.3), $n_{1}=-1, n_{2}=-1$, so $n_{1}+n_{2}+2=0$. Thus $u$ should be continuous across the dashed line in Fig. 2 but could have a singularity in its first derivative there. This is exactly the case. In the case depicted in Fig. 3, we see that if $u$ has order -1 at $x_{1}$ and $x_{2}$, then $u$ will have order at least $k-1$ across the lines $S_{k}$. Less precise results expressing the second general principle can be found in [1], [2], and [3]. 
The results of Sect. 3 guarantee that when anomalous singularities are produced they are weaker than the singularities which produce them. However, this does not answer the question of when the anomalous singularities actually exist. That is, which of the lines in $S$ are actually part of the singular support of $u$ and what is the order of $u$ across these lines? This is the question which is investigated in Sect. 4. To see that this is a delicate question, notice that if one looks at Fig. 2 and runs time backwards, then the singularity along the dashed lines disappears. We show that if $n_{1} \geqq 0, n_{2} \geqq 0, n_{i} \geqq n_{1}+n_{2}+2$, for $i \neq 1,2$, and if

$$
\frac{\partial f_{i}}{\partial u_{1} \partial u_{2}}(q, u(q)) \neq 0
$$

for some $i \neq 1,2$, where $q$ is the intersection point of $\mathscr{C}_{1}$ and $\mathscr{C}_{2}$, then $u$ will have order exactly $n_{1}+n_{2}+2$ on the $i$ th forward characteristic from $q$. This criterion permits us to construct examples where the anomalous singularities occur on all the isolated lines of $S$ with orders exactly equal to those predicted by the amelioration formula $m_{i}=\min \left\{n_{i}, n_{1}+n_{2}+2\right\}$. The analysis also explains the disappearance of singularities mentioned above.

The assumption of strict hyperbolicity guarantees that the $\left\{\lambda_{i}\right\}_{i=1}^{m}$ are distinct. In fact, the results of Sects. 2 and 3 go over virtually unchanged to the case where the multiplicities of the $\lambda_{i}$ are constant. It is merely necessary to regard the components of $u$ which correspond to the same speed $\lambda_{i}$ as a single vector-valued component. The ideas of the proofs in Sect. 4 also carry over easily to the case of constant multiplicity, but the main condition (4.1) is replaced by a matrix condition.

The elementary methods of the paper are useful for studying jump discontinuities across isolated curves, but they break down when the singular sets are more complicated. For example, suppose that the initial data are $H^{s}$ on an interval and $C^{\infty}$ outside of the interval. Then the union of forward cones (including the interiors) from the points in this interval will, in general, be the singular support of $u$ and this singular support should be stratified into regions of various degrees of smoothness because of the principle of amelioration of singularities. In order to handle these problems, one must construct a nonlinear microlocal calculus and apply it to semilinear hyperbolic systems. We do this in a forthcoming paper, [5]. That paper does not subsume the results of this one for two reasons. First, that paper deals only with the case of continuous functions so the interesting case of jumps in $u$ is excluded. Secondly, it is difficult to identify and study piece-wise smooth functions by the methods of Fourier analysis.

Although we have mentioned the solid forward cones, we don't prove anything about them in this paper. A natural extension of the results of Sect. 3 suggests that each time singularities intersect, the anomalous singularities produced should be at least one derivative smoother. Thus, if we are only interested in $C^{N}$ smoothness for fixed $N$, we should only have to consider finitely many interactions. So, if the data is $C^{\infty}$ except for finitely many jump discontinuities, we expect that the solution will be $C^{N}$ except on a finite network of curves. As $N$ gets larger, the number of singularity carrying curves increases, until, as $N \rightarrow \infty$, the curves become dense in the solid forward cones discussed above. We prove these statements in 
[5] and provide examples where the initial data is $C^{\infty}$ except for two points, but an entire solid forward cone is contained in the singular support of the solution.

Throughout this paper the symbol $[h]_{p}^{(i)}$ will denote the jump in the function $h$ at the point $p$ across the $i$ th member of a family of curves. For vertical curves the jump is from left to right; otherwise it is from bottom to top.

It is a pleasure to thank M. Oberguggenberger for helpful discussions.

\section{Smoothness}

In this section we shall prove that $u$ is $C^{\infty}$ on $R_{T} \backslash S$. If $\langle x, t\rangle$ is a point of $R_{T} \backslash S$, then the backward "cone" from $\langle x, t\rangle$ (i.e. the domain of dependence of $\langle x, t\rangle$ ) contains only finitely many curves, $\Gamma_{i}, i=1, \ldots, k$, from the set $S$. To see this, note that if the cone contained infinitely many intersection points of curves in $S$, then the closed forward cones from the limit points would contain $\langle x, t\rangle$ and would be contained in $S$. Since $\langle x, t\rangle \notin S$ the cone can contain only finitely many intersection points. Thus, by the definition of $S$, the only points of the cone which are also in $S$ are points in $S_{0}$ and the points on the forward characteristics from these finitely many intersection points. Since every $\left\langle x_{0}, t_{0}\right\rangle$ in $R_{T} \backslash S$ is contained in the interior of a backward cone, it suffices to prove the theorem in the case where $S \subset R_{T}$ contains only finitely many curves $\Gamma_{i}, i=1, \ldots, k$, and $R_{T}$ is a backward cone. We may also assume without loss of generality that the endpoints $a, b$ of the initial interval $I_{0}$ are not in the singular support of $u^{(0)}$. Thus $S$ divides $R_{T}$ up into finitely many simply connected open sets $R_{i}$ bounded by pieces of curves $\Gamma_{i}$ in $S$ and the boundaries of $R_{T}$; see Fig. 6 . The basic space which we will work with is the set of $R^{m}$-valued functions on $R_{T} \backslash S$ which are $N$ times continuously differentiable on $R_{T} \backslash S$ and such that all the first $N$ partial derivatives have continuous extensions from each of the $R_{i}$ to their boundaries (of course the boundary values on the curves $\Gamma_{i}$ will, in general, be different if one approaches $\Gamma_{i}$ from two different regions). We denote this space by $C_{*}^{N}\left(R_{T} \backslash S\right)$. Since $S$ will remain fixed from now on we will sometimes denote this space simply by $C_{*}^{N}\left(R_{T}\right)$; it is a Banach space under the norm

$$
\|u\|_{N}=\sum_{i}\|u\|_{C^{N}\left(R_{i}\right)}
$$

where $\|u\|_{C^{N\left(R_{i}\right)}}$ is the sum of the sups in $R_{i}$ of all partial derivatives of order less than or equal to $N$.

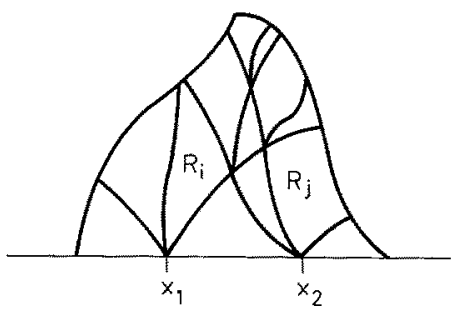

Fig. 6. The region $R_{T}$ 
Before proving the singular support theorem, we first derive a basic computational tool: a formula for differentiating certain indefinite line integrals of functions in $C_{*}^{N}\left(R_{T}\right)$. Let $R_{T}$ denote a region such as the one discussed above; we will denote the variables by $\langle y, s\rangle$. Suppose that $g \in C_{*}^{N}\left(R_{T}\right)$, and define

$$
u(y, s)=\int_{0}^{s} g(y, r) d r .
$$

Assume that the line $L_{y_{0}}=\left\{\left\langle y_{0}, r\right\rangle \mid 0 \leqq r \leqq s_{0}\right\}$ intersects the curves $\left\{\Gamma_{i}\right\}_{i=1}^{k}$ one at a time and transversally and that $\left\langle y_{0}, s_{0}\right\rangle \notin \cup \Gamma_{i^{*}}$. We want to prove that $u$ is $N$ times continuously differentiable and derive a formula for the derivatives. Since the $\Gamma_{i}$ are $C^{\infty}$ curves which are transverse to $L$, they are the graphs of $C^{\infty}$ functions $\gamma_{i}$ for $y$ near $y_{0}$. Relabeling the $\Gamma_{i}$ so that $\gamma_{i}<\gamma_{i+1}$, we can write

$$
u(y, s)=\int_{\gamma_{k}(y)}^{s} g(y, r) d r+\sum_{i=1}^{k} \int_{\gamma_{i-1}(y)}^{\gamma_{i}(y)} g(y, r) d r
$$

where we set $\gamma_{0}(y) \equiv 0$. Clearly, $\partial_{s} u$ exists and equals $g(y, s)$ for $\langle y, s\rangle$ near $\left\langle y_{0}, s_{0}\right\rangle$ and from the hypotheses on $g$ we get immediately that $\partial_{y}^{\ell_{1}} \partial_{s}^{\ell_{2}} u$ exists and is continuous near $\left\langle y_{0}, s_{0}\right\rangle$ if $\ell_{2} \geqq 1$ and $\ell_{1}+\ell_{2} \leqq N$. Thus, we need only consider the pure $y$ derivatives of $u$.

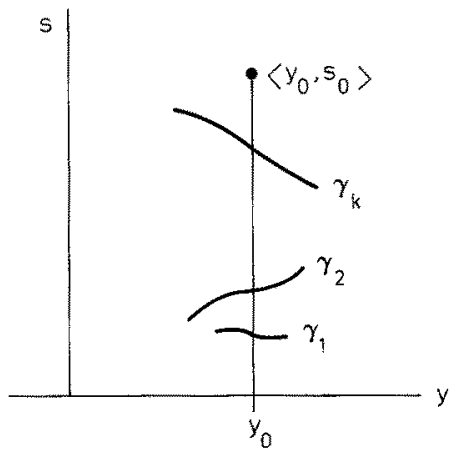

Fig. 7

Each term in (2.1) is continuously differentiable with respect to $y$. Differentiating and collecting terms, we find:

$$
\partial_{y} u(y, s)=\int_{0}^{s} \partial_{y} g(y, r) d r-\sum_{i=1}^{k}\left[\gamma_{i}^{\prime} g\right]_{\left\langle y, y_{i}(y)\right\rangle}^{(i)}
$$

where $\left[{ }^{\cdot}\right]_{p}^{(i)}$ denotes the jump from below $\Gamma_{i}$ to above $\Gamma_{i}$ at $p$. The integral is to be understood as a sum of integrals over the pieces of $L_{y}$. Because of the hypotheses on $g, \partial_{y} u$ is continuous so $u$ is continuously differentiable near $\left\langle y_{0}, s_{0}\right\rangle$.

To take the $\partial_{y}$ derivative of $\partial_{y} u$, notice that $\int_{0}^{s} \partial_{y} g(y, r) d r$ has the same form as the expression which we differentiated above, so

$$
\partial_{y} \int_{0}^{s} \partial_{y} g(y, r) d r=\int_{0}^{s} \partial_{y}^{2} g(y, r) d r-\sum_{i=1}^{k} \gamma_{i}^{\prime}(y)\left[\partial_{y} g\right]_{\left\langle y, \gamma_{i}(y)\right\rangle}^{(i)}
$$


Furthermore, $\left[\gamma_{i}^{\prime} g\right]_{\left\langle y, \gamma_{i}(y)\right\rangle}^{(i)}=\gamma_{i}^{\prime}(y)\left\{g^{+}\left(y, \gamma_{i}(y)\right)-g^{-}\left(y, \gamma_{i}(y)\right)\right\}$ is differentiable in $y$ and

$$
\partial_{y}\left[\gamma_{i}^{\prime} g\right]_{\left\langle y, \gamma_{i}(y)\right\rangle}^{(i)}=\left[\left(\partial_{y}+\gamma_{i}^{\prime} \partial_{s}\right) \gamma_{i}^{\prime} g\right]_{\left\langle y, y_{i}(y)\right\rangle}^{(i)}
$$

Therefore,

$$
\begin{aligned}
\partial_{y}^{2} u=\int_{0}^{s} \partial_{y}^{2} g(y, r) d r & -\sum_{i=1}^{k}\left[\gamma_{i}^{\prime} \partial_{y} g\right]_{\left\langle y, y_{i}(y)\right\rangle}^{(i)} \\
& -\sum_{i=1}^{k}\left[\left(\partial_{y}+\gamma_{i}^{\prime} \partial_{s}\right) \gamma_{i}^{\prime} g\right]_{\left\langle y, \gamma_{i}(y)\right\rangle}^{(i)} .
\end{aligned}
$$

It is clear that we can continue in this way differentiating $N$ times with respect to $y$ and obtain, for $n \leqq N$ :

$$
\partial_{y}^{n} u(y, s)=\int_{0}^{s} \partial_{y}^{n} g(y, r) d r-\sum_{i=1}^{k} \sum_{\ell=0}^{n-1}\left[\left(\partial_{y}+\gamma_{i}^{\prime} \hat{o}_{s}\right)^{\ell} \gamma_{i}^{\prime} \partial_{y}^{n-1-\ell} g\right]_{\left\langle y, \gamma_{i}(y)\right\rangle}^{(i)}
$$

where $[\cdot]_{\left\langle y, \gamma_{i}(y)\right\rangle}^{(i)}$ denotes the jump from below $\Gamma_{i}$ to above at $\left\langle y, \gamma_{i}(y)\right\rangle$. Thus $u$ is $C^{N}$ at $\left\langle y_{0}, s_{0}\right\rangle$.

Notice that the answer in (2.4) is independent of the ordering of $\left\{\gamma_{i}\right\}$. We also remark that our notation seems to imply that the line $L_{y_{0}}$ intersects each curve $\Gamma_{i}$ only once. We need no such hypothesis since the analysis is completely local in a neighborhood of $L$. If one of the $\Gamma_{i}$ interests $L_{y_{0}}$ more than once, we just treat the pieces of $\Gamma_{i}$ near the different intersection points as separate curves.

We summarize these computations in a lemma:

Lemma 2.1. Let $g$ be a function from a neighborhood, $\mathscr{N}$, of the line $L_{y_{0}}=\left\{\left\langle y_{0}, r\right\rangle\right.$ $\left.\mid 0<r<s_{0}\right\}$ to $\mathbb{R}^{m}$ such that $g$ is $C^{N}$ except across finitely many $C^{N}$ curves $\Gamma_{i}, i=1, \ldots, k$, which intersect $L_{y_{0}}$ transversally. Suppose that $\left\langle y_{0}, s_{0}\right\rangle$ is not on any $\Gamma_{i}$ and that $L_{y_{0}}$ intersects the $\Gamma_{i}$ one at a time (i.e. $L_{y_{0}} \cap \Gamma_{i} \cap \Gamma_{j}=\varnothing$ if $i \neq j$ ). Suppose that all the derivatives of $g$ up to order $N$ have, for each $i,($ local $)$ continuous extensions from each side of $\Gamma_{i}$ up to $\Gamma_{i}$ and define $u$ in a neighborhood of $\left\langle y_{0}, s_{0}\right\rangle$ by

$$
u(y, s)=\int_{0}^{s} g(y, r) d r .
$$

Then $u$ is $C^{N}$ in a neighborhood of $\left\langle y_{0}, s_{0}\right\rangle$ and the $y$ derivatives satisfy (2.4).

We are now ready for the main theorem:

Theorem 1. Let $R_{T}$ be a region of $\mathbb{R}^{2}$ as described in the introduction with initial interval $I_{0}=[a, b]$ and suppose that $\Lambda$ is $C^{\infty}$ in a neighborhood $\mathscr{N}$ of $\bar{R}_{T}$ and that $F: \mathcal{N} \times \mathbb{R}^{m} \rightarrow \mathbb{R}^{m}$ is $C^{\infty}$. Let $u \in L^{\infty}\left(R_{T}\right)^{m}$ satisfy (1.2) in the sense of distributions and take on the initial data $u^{0}(x) \in L^{\infty}\left(I_{0}\right)$. Suppose that $u^{0}(x)$ is $C^{\infty}$ with each derivative uniformly bounded on the complement of finitely many points $\left\{x_{i}\right\}_{i=1}^{\ell}$. Let $S$ be the closed subset of $R_{T}$ defined in the introduction.

Then $u$ is $C^{\infty}$ on $R_{T} \backslash S$ and all derivatives of $u$ have continuous extensions from each connected component of $R_{T} \backslash S$ to its closure.

Proof. As described at the beginning of this section, it is sufficient to consider the case where $R_{T}$ is a backward cone and $S$ consists of finitely many curves. We will 
show that for each integer $N \geqq 0, u$ is in $C_{*}^{N}\left(R_{T} \backslash S\right)$. Since the initial value problem (1.2) has at most one solution in $L^{\infty}\left(R_{T}\right)$, it suffices to show that there is a solution in $C_{*}^{N}\left(R_{T} \backslash S\right)$, for this solution must be $u$. We construct the solution in $C_{*}^{N}$ by solving the integral equations corresponding to (1.2).

Suppose that $w \in C_{*}^{N}\left(R_{t_{0}} \mid S\right)$ and, for $\langle x, t\rangle \in R_{t_{0}}$, define

$$
(\mathscr{H} w)_{i}(x, t)=u_{i}^{o}(\xi(0))+\int_{\mathscr{C}_{i}(x, t)} f_{i}(\xi(a), w(\xi(a)))\left(1+\lambda_{i}(\xi(a))^{2}\right)^{1 / 2} d a
$$

where $\mathscr{C}_{i}(x, t)$ is the $i$ th backward characteristic from $\langle x, t\rangle$ to $I_{0}$ and $a \rightarrow \xi(a)$ is the arclength parametrization of $\mathscr{C}_{i}(x, t)$. If $w$ is in $C_{*}^{N}$ and $\mathscr{M} w=w$, then $w$ satisfies (1.2) in the sense of distributions and takes on the correct initial data. The crucial step in finding a fixed point in $C_{*}^{N}$ is to show that $\mathscr{M}$ takes $C_{*}^{N}\left(R_{t_{0}} \backslash S\right)$ into itself. Suppose that $\langle x, t\rangle \notin S$. Then $\mathscr{C}_{i}(x, t)$ intersects the curves in $S$ (which we denote by $\left\{\Gamma_{j}\right\}$ one at a time, for if $\mathscr{C}_{i}(x, t)$ intersected an intersection point of two or more of the $\Gamma_{j}$ then $\langle x, t\rangle$ would be in $S$ by the way in which $S$ was defined. For such $\langle x, t\rangle \notin S$, we want to show that $(\mathscr{M} w)_{i}$ is $N$ times continuously differentiable. In order to do this it is convenient to work in coordinates so that the $i$ th vector field, $\partial_{t}+\lambda_{i} \partial_{x}$, is straightened out. Define a mapping $\eta_{i}:\langle x, t\rangle \rightarrow\langle y, s\rangle$ by letting $y$ be the $x$-coordinate of the point where $\mathscr{C}_{i}(x, t)$ intersects $I_{0}$ and $s$ be the length of $\mathscr{C}_{i}(x, t)$ from $\langle y, 0\rangle$ to $\langle x, t\rangle$. Because $A(x, t)$ is $C^{\infty}, \eta_{i}$ is a $C^{\infty}$ diffeomorphism on all of $R_{T}$. Denote by $\Gamma_{j}$ the characteristic curves of the jth family in the new coordinates. In these coordinates the $i$ th family becomes a collection of vertical straight lines $L=\{\langle y, r\rangle \mid 0 \leqq r \leqq s(y)\}$. The strict hyperbolicity of (1.1) implies that the $\Gamma_{j}$ intersect $L$ transversally for $j \neq i$. Thus, the $\Gamma_{j}$ satisfy the hypotheses of Lemma 2.1. In the new coordinates $\langle y, s\rangle$ we have

$$
(\mathscr{A} w)_{i}(y, s)=u_{i}^{0}(y)+\int_{0}^{s} f_{i}(y, r, w(y, r)) d r .
$$

Since $w \in C_{*}^{N}\left(R_{t_{0}} \backslash S\right)$ and $f_{i}$ is $C^{\infty}, g=f_{i}(y, r, w(y, r))$ satisfies the hypotheses of Lemma 2.1 too. Since $\langle y, s\rangle \notin S,\langle y, 0\rangle$ is not in the singular support of the initial data. Thus, by Lemma $2.1,(\mathscr{M} w)_{i}$ is $C^{N}$ near $\langle y, s\rangle$. Furthermore, since all of the objects that appear in the explicit differentiation formula (2.4) are functions continuous up to the $\Gamma_{i}$ or indefinite integrals of such functions, it is easy to see that all the derivatives of $(\mathscr{M} w)_{i}$ up to order $N$ are continuous up to the $\Gamma_{i}$. Thus $\mathscr{M} w \in C_{*}^{N}\left(R_{t_{0}} \backslash S\right)$ if $w \in C_{*}^{N}\left(R_{t_{0}} \backslash S\right)$.

To solve $\mathscr{M}_{w}=w$, one shows that for $t_{0}$ small $\mathscr{M}$ is a contraction on a ball in $C_{*}^{N}\left(R_{t_{0}} \backslash S\right)$ if the norm is suitably modified. This type of argument is relatively straight-forward, so we just provide a sketch. Define

$$
\|u\|_{k, t_{0}}=\sum_{i=1}^{m} \sum_{\ell=0}^{k} \sup _{\langle x, t\rangle \in R_{t_{0}} \mid S}\left|\left(\frac{\partial}{\partial x}\right)^{\ell}\left(\frac{\partial}{\partial t}\right)^{k-\ell} u_{i}(x, t)\right| .
$$

We norm $C_{*}^{N}\left(R_{t_{0}} \backslash S\right)$ by

$$
\|u\|_{t_{0}}^{(N)}=\sum_{j=0}^{N} \varepsilon_{j}\|u\|_{j, t_{0}}
$$

where the $\varepsilon_{0}=1$, and the other $\varepsilon_{j}$ are small positive constants (depending on $F$ ) 
to be chosen shortly. We denote by $X_{t_{0}}^{N}$ the set of $w$ in $C_{*}^{N}\left(R_{t_{0}} \mid S\right)$ such that $w(x, 0)=$ $u^{0}(x, 0)$ and $\|w\|_{t_{0}}^{(N)} \leqq 2\left\|u_{0}\right\|_{t_{0}}^{(N)}$ where $u_{0}(x, t)$ is the solution of $(1.2)$ if $F \equiv 0$. To estimate the derivatives of $(\mathscr{M} w)_{i}$ we use the coordinate system $\left\langle y^{i}, s^{i}\right\rangle$ given by the $C^{\infty}$ diffeomorphism $\eta_{i}$. The derivatives in the original coordinate system are then estimated by the $\left\langle y^{i}, s^{i}\right\rangle$ derivatives times some $\eta_{i}$-dependent factors. Since the $\eta_{i}$ depend only on $A$, these factors have universal bounds in terms of $\|A\|_{C^{N}\left(R_{T}\right)}$. For $N=0$, the contraction proof is standard and we get a solution in $X_{t_{0}}^{0}$ for $t_{0}$ small enough. For $N=1$, there will be terms in the expressions for $\frac{\partial}{\partial x}(\mathscr{M} w-\mathscr{M} \tilde{w})$ and $\frac{\partial}{\partial t}(\mathscr{M} w-\mathscr{M} \tilde{w})$ which involve $f_{i}(., w(\cdot))$ evaluated at points. Since $f_{i}$ is Lipschitz on bounded sets, there is a $C$ such that for all $w, \tilde{w} \in X_{t_{0}}^{0}$ and $t_{1} \leqq t_{0}$, we have:

$$
\left\|f_{i}(\cdot, w(\cdot))-f(\cdot \tilde{w}(\cdot))\right\|_{t_{1}}^{0}<C\|w-\tilde{w}\|_{t_{1}}^{0} .
$$

Since $C$ is not small at looks hard to prove the contraction property. This is the reason for $\varepsilon_{1}$. By choosing the norm as we did, when we compute $\|\mathscr{M} w-\mathscr{M} \tilde{w}\|_{t_{1}}^{1}$ there is an additional factor of $\varepsilon_{1}$ on both sides of (2.5). $\varepsilon_{1}$ is just chosen so that $\varepsilon_{1} C<1$. With this trick it is easy to prove that $\mathscr{M}$ is a contraction on $X_{t_{1}}^{1}$ for $t_{1}$ small (in general smaller than $t_{0}$ ).

The proofs for higher $N$ are similar. Notice, however, that as $N \rightarrow \infty$ we may have $t_{N} \rightarrow 0$. We would like to fix $N$ and apply the above local existence theorem and show that after finitely many applications we will have $u \in C_{*}^{N}\left(R_{T} \backslash S\right)$. There are two problems. The first is that we must show that the interval of existence does not shrink to zero. We do this by proving an apriori estimate which shows that for $0<\tilde{t}<T$ and $u$ in $X_{\tilde{t}}^{N}$ satisfying the differential equation,

$$
M_{N}(\tilde{t})=\sum_{i=1}^{m} \sum_{k=0}^{N} \sum_{\ell=0}^{k} \sup _{R_{\tilde{t}} \leq}\left|\partial_{x}^{\ell} \partial_{t}^{k-\ell} u_{i}(x, t)\right|
$$

has a bound independent of $\tilde{t}$. This is accomplished as follows: We bound the first derivatives of $u_{i}$ using the $\left\langle y^{i}, s^{i}\right\rangle$ coordinates. Since $\partial_{s} u_{i}=f_{i}(u)$, the bound on $u$ yields a bound on $\partial_{s} u_{i}$. To bound $\partial_{y} u_{i}$, we use the differentiation formula (2.4) to obtain

$$
\left.\left(\partial_{y} u_{i}\right)(y, s)=\int_{0}^{s} \partial_{y} f_{i}(u(y, r)) d r+\partial_{y} u_{i}(y, 0)-\sum_{j=1}^{k}\left[\gamma_{j}^{\prime} f_{i}\right]_{\left\langle y, \gamma_{j}(y)\right\rangle}^{(j)}\right\rangle
$$

If $\langle y, s\rangle \notin S$, then $u_{i}(\cdot, 0)=u_{i}^{0}($.$) is smooth at y$ and, by the hypotheses on $u^{0},\left|\partial_{y} u_{i}^{0}\right|$ is uniformly bounded for such $y$. The third term depends only on $u$ and is thus bounded by a constant depending only on $M_{0}$ (we will denote constants depending only on $T, A, F$ and $M_{0}$ by $\left.C\left(M_{0}\right)\right)$. Thus,

$$
\begin{aligned}
\left|\partial_{y} u_{i}(y, s)\right| & \leqq C\left(M_{0}\right)+C\left(M_{0}\right) \int_{0}^{s}\left|\left(\partial_{y} f_{i}\right)(y, r, u)+\sum_{j=1}^{m}\left(\partial_{u_{j}} f_{i}\right)\left(\partial_{y} u_{j}(y, r)\right)\right| d r \\
& \leqq C\left(M_{0}\right)+C\left(M_{0}\right) \int_{0}^{s} \sum_{j=1}^{m}\left|\partial_{y} u_{j}(y, r)\right| d r .
\end{aligned}
$$


Returning to the original variables, we find that

$$
M_{1}(t) \leqq C\left(M_{0}\right)+C\left(M_{0}\right) \int_{0}^{t} M_{1}(p) d p
$$

which by Gronwall's inequality shows that $M_{1}(t)$ is apriori bounded in $R_{\tilde{t}}$ by a constant $M_{1}$.

We now use the same procedure to estimate $M_{2}(t)$ and so forth. The two crucial points are, first, that the jump terms in the differentiation formula (2.4) for the $(N+1)^{s t}$ derivatives depend only on the first $N$ derivatives and thus are bounded by the induction hypothesis. Secondly, the term under the integral in the formulas is always linear in the $N+1^{\text {st }}$ derivatives and so, when the lower order terms are dominated by the induction hypothesis, the Gronwall lemma again gives the result for $N+1$. Thus each $M_{n}(t)$ is apriori bounded so we can continue to apply the local existence theorem and arrive at $T$ after finitely many steps.

There is one last difficulty. The local existence theorem gives us a solution in $R_{t_{0}}$. This solution will be singular, at most, at the points $\left\{z_{i}\right\}_{i=1}^{h}$ where the line $t=t_{0}$ intersects $S$. If $z_{i}$ is an intersection of two or more curves in $S$, then all forward characteristics from $z_{i}$ are in $S$ and there is no problem. If $z_{i}$ is on only one curve, $G_{\ell}$ in $S$, then we expect (and are trying to prove) that near $t_{0}$ the singularity will continue on the curve and not go on the other forward characteristic curves (let's call them $\left\{G_{i} \mid i \neq \ell\right\}$ from $z_{i}$. Unfortunately, from the local existence result starting at $t_{0}$, we can only conclude that $u$ is $C^{N}$ away from the union of the curves in $S$ and the $\left\{G_{k}\right\}$. This difficulty is overcome as follows: If we apply the local existence result with initial data $u\left(x, t_{0}-\varepsilon\right)$ given at time $t^{\prime}=t_{0}-\varepsilon$ then we conclude that $u$ is $C^{N}$ near $z_{i}$ except on the union of $S$ and the other forward characteristics $\left\{G_{k}^{\prime}\right\}$ from the singular point $z_{i}^{\prime}$. Since the $\lambda_{i}$ are distinct, the $\left\{G_{k}^{\prime}\right\}$ can intersect the $\left\{G_{i} \mid i \neq \ell\right\}$ in only finite many points such as $p$ in Fig. 8. We conclude that $u$ is $C^{N}$ across the $\left\{G_{i} \mid i \neq \ell\right\}$ except possibly for these finitely many points. By varying $\varepsilon$, we can eliminate these points also.

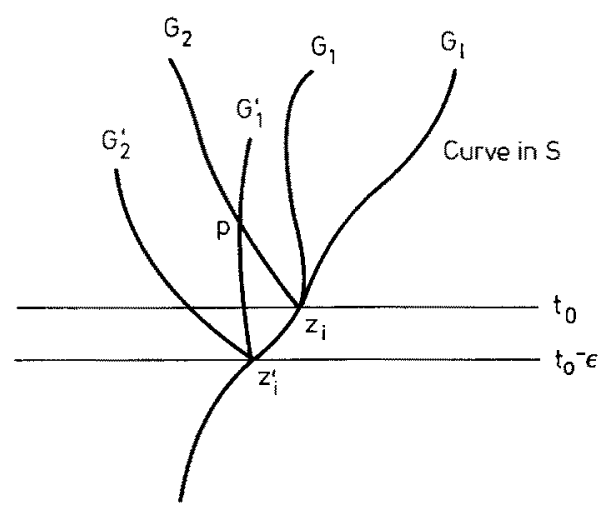

Fig. 8. A neighborhood of $z_{i}$ 


\section{Interaction of Singularities}

In this section we derive an upper bound for the strengths of the anomalous singularities produced when two or more singularities meet. Let $q$ be a point of intersection of isolated curves in $S$ and let $R$ be a neighborhood of $q$ of the form described in the introduction (see Fig. 9) so that there are no other intersection points in $R$. By a change of time variable, we may label the beginning, the intersecting, and the ending times of $R$ by $t=0, \frac{1}{2}$, and 1 , respectively. Lable the characteristics through $q$ by $\mathscr{C}_{i}, i=1, \ldots, m$, and let $n_{i}, i=1, \ldots, m$ be the largest integer so that $u$ is $C^{n_{i}}$ across $\mathscr{C}_{i}$ for $t \in\left[0, \frac{1}{2}\right)$, i.e. so that $u$ is in $C^{n_{i}}$ in a neighborhood of the incoming half of $\mathscr{C}_{i}$. We set $n_{i}=\infty$ if $u$ is $C^{\infty}$ across $\mathscr{C}_{i}$ for $t \in\left[0, \frac{1}{2}\right.$ ). If $u$ itself jumps, $n_{i}$ will equal $-1 ; n_{i}$ will equal 0 if $u$ is continuous but a first derivative jumps, and so forth. We relable the characteristics if necessary so that $-1 \leqq n_{1} \leqq n_{2} \leqq n_{i}$ if $i \neq 1,2$. There are two trivial cases. If $n_{1}=n_{2}=\infty$ (which is possible since $S$ contains but does not necessarily equal sing supp $u$ ), then $u$ is $C^{\infty}$ in $R$ for $t \in\left[0, \frac{1}{2}\right)$ and Theorem 1 implies that $u$ is $C^{\infty}$ in all of $R$. If $n_{1}<\infty$ and $n_{2}=\infty$, then Theorem 1 applied at time $t=\frac{1}{4}$ together with the argument at the very end of the proof of Theorem 1 show that $u$ is $C^{\infty}$ in $R$ except on $\mathscr{C}_{1}$ across which it is at least $C^{n_{1}}$. This just repeats what we already know, namely that anomalous singularities can only be produced when two or more singularity bearing characteristics intersect.

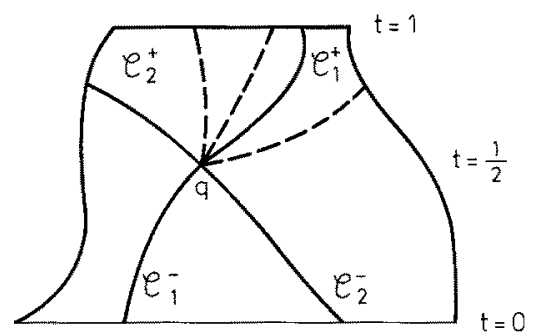

Fig. 9. The region $R$

When $n_{2}<\infty$, we will show that $u$ is $C^{m_{i}}$ across $\mathscr{C}_{i}$ for $t \in\left(\frac{1}{2}, 1\right]$ where

$$
m_{i}=\min \left\{n_{i}, n_{1}+n_{2}+2\right\} .
$$

The usual case is when the curves in $S$ intersect two at a time, i.e. $n_{i}=\infty$ for $i \neq 1,2$. In this case $u$ is at least $C^{n_{1}+n_{2}+2}$ across the forward characteristics from $q$ except $\mathscr{C}_{1}$ and $\mathscr{C}_{2}$. If one of the other incoming characteristics, $\mathscr{C}_{i}$, is carrying a singularity, i.e. $n_{i}<\infty$ for some $\mathrm{i} \neq 1,2$, then we would not expect $u$ to be better than $C^{n_{i}}$ on the outgoing part of $\mathscr{C}_{i}$ and this is reflected by the formula (3.1).

The proof consists of several steps which gradually establish more and more smoothness across the outgoing parts of the $\mathscr{C}_{i}$. First we show that $u$ is $C^{n_{1}}$ in $R$. Then we prove that, for $t \in\left(\frac{1}{2}, 1\right], u$ is $\mathscr{C}^{n_{2}}$ across all $\mathscr{C}_{i}$ except $i=1$. Then we prove a proposition which gives conditions that guarantee that if the jump $\left[D^{\ell} u\right]^{(i)}$ is continuous at $q$ then so is the jump $\left[D^{\ell} h(u)\right]^{(i)}$ for any $h \in C^{\infty}$ provided $|\ell| \leqq n_{1}+n_{2}+1$. This is the origin of the number $n_{1}+n_{2}+2$. Then we prove that 
the jumps $\left[D^{\ell} u\right]^{(i)}$ are continuous at $q$ for $|\ell| \leqq n_{1}+n_{2}+2$. Finally, we prove the main theorem.

Throughout the proofs, $D^{\ell}$ denotes an $\ell$ th order partial derivative and $\left[D^{\ell} u\right]_{p}^{(i)}$ denotes the jump at $p$ across the $i$ th characteristic curve $\mathscr{C}_{i}$ through $q$.

Step 1. $u$ is $C^{n_{1}}$ in $R$

Proof. If $n_{1}=-1$ we have nothing to prove since we already know that $u$ is in $C_{*}^{\infty}$. If $n_{1} \geqq 0$, then standard regularity theorems in one space variable (or one can use the proof of Theorem 1) show that $u$ is $C^{n_{1}}$ since the data (at $t=0$ ) is in $C^{n_{1}}$.

Recall that if $E$ is a finite set of $\operatorname{arcs}$ in $R$, then $C_{*}^{k}(R \backslash E)$ is the set of functions $v \in C^{k}(R \backslash E)$ such that for each component $R_{i}$ of $R \backslash E$ and each $|\ell| \leqq k, D^{\ell} v\left\lceil R_{i}\right.$ has a continuous extension to $\bar{R}_{i}$.

Step 2. $u$ is in $C_{*}^{n_{2}}\left(R \backslash \mathscr{C}_{1}\right)$

Proof. If $n_{1}=n_{2}=-1$, there is nothing to prove so we may assume that $n_{2} \geqq 0$. We will prove that if $u \in C_{*}^{k}\left(R \backslash \mathscr{C}_{1}\right)$ and $k+1 \leqq n_{2}$, then $u \in C_{*}^{k+1}\left(R \backslash \mathscr{C}_{1}\right)$. Since, by Step 1 , the hypothesis is valid for $k=n_{1}$, this suffices to establish the result. To show that $u \in C_{*}^{k+1}\left(R \backslash \mathscr{C}_{1}\right)$, we will first show that for any $p \notin \mathscr{C}_{1}, u$ is in $C^{k+1}$ on a neighborhood of $p$. Since $u \in C_{*}^{\infty}(R \backslash S)$ by Theorem 1 , it suffices to consider $p \in \mathscr{C}_{i} \backslash\{q\}$ for $i \neq 1$.

First suppose $j \neq i$. We work in the variables $\left\langle y^{j}, s^{j}\right\rangle$ given by the diffeomorphism $\eta_{j}, D^{k+1} u_{j}=\partial_{y}^{\ell_{1}} \partial_{s} u_{j}(y, s)$ where $\ell_{1}+\ell_{2}=k+1$. If $\ell_{2} \geqq 1$, then $D^{k+1} u_{j}=$ $\partial_{y}^{\ell_{1}} \partial^{\ell_{2}-1} f_{j}(u)$ so $D^{k+1} u_{j}$ is in $C_{*}\left(R \backslash \mathscr{C}_{1}\right)$ by the induction hypothesis. Thus, we need only consider $\partial_{y}^{k+1} u_{j}$. Let $p_{0}=\left\langle y_{0}, s_{0}\right\rangle$ be a point on $\mathscr{C}_{i}, p_{0} \neq q$. According to the differentiation formula (2.4),

$$
\begin{aligned}
\partial_{y}^{k+1} u_{j}\left(y_{0}, s_{0}+\varepsilon\right)-\partial_{y}^{k+1} u_{j}\left(y_{0}, s_{0}-\varepsilon\right)= & \int_{s_{0}-\varepsilon}^{s_{0}+\varepsilon} \partial_{y}^{k+1} f_{j}(u(y, r)) d r \\
& + \text { jump terms at } p_{0} .
\end{aligned}
$$

But, the jump terms at $p_{0}$ are all zero by the induction hypothesis since they involve only derivatives of order at most $k$. Since $\partial_{y}^{k+1} f_{j}(u)$ is bounded, it follows that $\partial_{y}^{k+1} u_{j}$ is continuous across $\mathscr{C}_{i}$.

To handle the component $u_{i}$, we work in the variables $\left\langle y^{i}, s^{i}\right\rangle$ given by $\eta_{i}$. As above, the induction hypothesis immediately handles all partials of order $k+1$ except $\partial_{y}^{k+1} u_{i}$. Let $\left\langle y_{0}, s_{0}\right\rangle$ be a point on $\mathscr{C}_{i}$ for $t>\frac{1}{2}$. For $y$ near $y_{0}$, formula (2.4) yields

$$
\begin{aligned}
\partial_{y}^{k+1} u_{i}\left(y, s_{0}\right)= & \partial_{y}^{k+1} u_{i}(y, 0)+\int_{0}^{s_{0}} \partial_{y}^{k+1} f_{i}(u(y, r)) d r \\
& -\sum_{j \neq i} \sum_{\ell=0}^{k}\left[\left(\partial_{y}+\gamma_{j}^{\prime} \partial_{s}^{\ell} \gamma_{j}^{\prime} \partial_{y}^{k-\ell} f_{i}(u)\right]_{\left\langle y, \gamma_{j}(y)\right\rangle}^{(j)}\right.
\end{aligned}
$$

Since $k \leqq n_{2}-1, \partial_{y}^{k+1} u_{i}(y, 0)$ is continuous near $y=y_{0}$. By the induction hypothesis, all of the jump terms are zero except for $j=1$. On the other hand $f_{i}(u)$ is in $C_{*}^{k}\left(R \backslash \mathscr{C}_{1}\right)$ by the induction hypothesis, so each term $\left[\left(\partial_{y}+\gamma_{i}^{\prime} \partial_{s}\right)^{\ell} \gamma_{i}^{\prime} \partial_{y}^{k-\ell} f_{i}(u)\right]_{\left\langle\gamma, \gamma_{i}(\gamma)\right\rangle}^{(1)}$ 


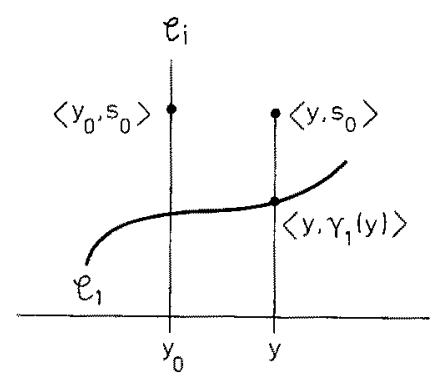

Fig. 10

is continuous for $y$ near $y_{0}$. Furthermore, $\partial_{y}^{k+1} f_{i}(u(y, r))$ contains many terms but all except the term $\left(\partial_{u_{i}} f_{i}\right)\left(\partial_{y}^{k+1} u_{i}\right)$ are continuous near $y=y_{0}$ since $u \in C_{*}^{k}\left(R \backslash \mathscr{C}_{1}\right)$ and for $j \neq i$, we have already shown that $u_{j} \in C_{*}^{k+1}\left(R \backslash \mathscr{C}_{1}\right)$. Thus, we may write (3.2) as

$$
\partial_{y}^{k+1} u_{i}\left(y, s_{0}\right)=c(y)+\int_{0}^{s_{0}}\left(\partial_{u_{i}} f_{i}\right)(u(y, r))\left(\partial_{y}^{k+1} u_{i}(y, r)\right) d r
$$

where $c(y)$ is continuous for $y$ near $y_{0}$. Substracting this expression for $y<y_{0}$ from this expression for $y>y_{0}$ and taking the limit as $y$ goes to $y_{0}$ we find:

$$
\left[\partial_{y}^{k+1} u_{i}\right]_{\left\langle y_{0}, s_{0}\right\rangle}^{(i)}=\int_{0}^{s_{0}}\left(\partial_{u_{i}} f_{i}\right)(u)\left[\partial_{y}^{k+1} u_{i}\right]_{\left\langle y_{0}, r\right\rangle}^{(i)} d r
$$

where []$^{(i)}$ denotes the jump from left to right. Since (3.4) holds for all $s_{0} \in[0,1]$ and $\left(\partial_{u_{i}} f_{i}\right)\left(u\left(y_{0}, r\right)\right)$ is bounded, Gronwall's inequality implies that $\left[\partial_{y}^{k+1} u_{i}\right]_{\left\langle y_{0}, s_{0}\right\rangle}^{(i)}=0$.

To complete the proof, we need only consider $u$ near $\mathscr{C}_{1}$. Consider the restriction of $u$ to one side, $\mathscr{S}^{+}$, of $\mathscr{C}_{1}$. We must show that $u\left\lceil\mathscr{S}^{+}\right.$has a $C^{k+1}$ extension to $\overline{\mathscr{S}}^{+}$. The first part of the proof together with the fact that $u \in C_{*}^{\infty}(R \backslash S)$ shows that $u$ has a $C^{k+1}$ extension to $\overline{\mathscr{S}}^{+} \backslash\{q\}$. In fact, $\{q\}$ is easily handled too. Notice that $\mathscr{S}^{+}$is decomposed by the $\mathscr{C}_{i}$ into $m$ triangular shaped regions $\mathscr{T}_{1}, \ldots, \mathscr{T}_{m}$. Suppose $|\ell|=k+1$ and let $h_{i}$ denote the continuous extension of $D^{\ell} u$ to $\mathscr{T}_{i}$ guaranteed by Theorem 1 . Then, $D^{\ell} u$ has a continuous extension to $\overline{\mathscr{S}}^{+}$if and only if $h_{i}(q)=h_{j}(q)$ for all $i, j$. We can label the $\mathscr{T}_{i}$ so that $\overline{\mathscr{T}}_{i} \cap \overline{\mathscr{T}}_{i+1}$ contains a characteristic arc, $\Gamma$, through $q$. From the first part of the proof we know that $h_{i}=h_{i+1}$ on $\Gamma \backslash\{q\}$ so, by the continuity of the $h_{i}$, it follows that $h_{i}(q)=h_{i+1}(q)$ and the proof is complete.

The third step is the proof of the following proposition. It asserts that certain regularity is preserved under non linear maps and explains the occurrence of the number $n_{1}+n_{2}+2$.

Proposition 3.1. Let $\left\{\mathscr{C}_{i}\right\}_{i=1}^{M}$ be $M$ smooth curves in $\mathbb{R}^{2}$ which intersect at a point $q$ where their slopes are distinct. Suppose that $\Omega$ is a neighborhood of $q$ which contains no other intersection points and let $v$ be a function on $\Omega$ satisfying:

(i) $v \in C_{*}^{\infty}\left(\Omega \backslash \bigcup_{i=1}^{M} \mathscr{C}_{i}\right)$ 
(ii) $v \in C^{n_{1}}(\Omega)$

(iii) $v \in C_{*}^{n_{2}}\left(\Omega \backslash \mathscr{C}_{1}\right)$

(iv) For some $i$ and some $k \leqq n_{1}+n_{2}+1,\left[D^{\ell} v\right]^{(i)}$ is continuous on $\mathscr{C}_{i}$ for all $|\ell| \leqq k$.

Then:

(v) $\left[D^{\ell} h(v)\right]^{(i)}$ is continuous on $\mathscr{C}_{i}$ for all $|\ell| \leqq k$ and all $C^{\infty}$ functions $h$.

Proof. When $n_{1}=-1$ the problem simplifies since $k \leqq n_{2}$ which implies, by (iii), that $v \in C_{*}^{k}\left(\Omega \backslash \mathscr{C}_{1}\right)$. Thus if $|\ell| \leqq k,\left[D^{\ell} h(v)\right]_{p}^{(i)} \equiv 0$ if $i \neq 1$, and $\left[D^{\ell} h(v)\right]_{p}^{(1)}$ is continuous.

Next, suppose that $n_{1} \geqq 0 . D^{\ell} h(v)$ is a sum of terms of the form

$$
\left(D^{\alpha} h\right)(v) \prod_{n} D^{\beta_{n}} v_{j(n)} \quad|\alpha| \leqq|\ell|, \quad \sum\left|\beta_{n}\right|=|\ell| .
$$

Since $[\cdot]_{p}^{(i)}$ is linear, it suffices to consider each of these terms separately. Also, since $n_{2} \geqq n_{1} \geqq 0,\left(D^{\alpha} h\right)(v)$ is continuous everywhere in $\Omega$. It thus suffices to consider $\left[\prod_{n} D^{\beta_{n}} v_{j(n)}\right]_{p}^{(i)}$. Since $\sum\left|\beta_{n}\right| \leqq n_{1}+n_{2}+1$ and $n_{1} \leqq n_{2}$, at most one of the $\left|\beta_{n}\right|$ can be bigger than $n_{2}$. Suppose that there is such a $\beta_{n}$, call it $\beta_{1}$. Then, we factor:

$$
\left[\prod_{n \geqq 1} D^{\beta_{n}} v_{j(n)}\right]_{p}^{(i)}=\left[D^{\beta_{t}} v_{j(1)}\right]_{p}^{(i)}\left(\prod_{n \geqq 2} D^{\beta_{n}} v_{j(n)}\left(p^{+}\right)\right)+\left(D^{\beta_{1}} v_{j(1)}\left(p^{-}\right)\right)\left[\prod_{n \geqq 2} D^{\beta_{n}} v_{j(n)}\right]_{p}^{(i)}
$$

where $p^{ \pm}$denote the limits at $p$ from the two sides of $\mathscr{C}_{i}$. For $n \geqq 2, D^{\beta_{n}} v_{j(n)}$ is continuous in $\Omega$ since $\left|\beta_{n}\right|<n_{1}$. Thus $\left[\prod_{n \geqq 2} D^{\beta_{n}} v_{j(n)}\right]_{p}^{(i)}=0$ and $\prod_{n \geqq 2} D^{\beta_{n}} v_{j(n)}\left(p^{+}\right)$is continuous. Since $\left[D^{\beta_{1}} v_{j(1)}\right]_{p}^{(i)}$ is continuous by hypothesis, we conclude that $\left[\prod_{n \geq 2} D^{\beta_{n}} v_{j(n)}\right]_{p}^{(i)}$ is continuous.

On the other hand, if all $\beta_{n}$ satisfy $\left|\beta_{n}\right| \leqq n_{2}$, then $\prod D^{\beta_{n}} v_{j(n)}$ is in $C_{*}^{0}\left(\Omega \backslash \mathscr{C}_{1}\right)$. Thus $\left[\prod_{n} D^{\beta_{n}} v_{j(n)}\right]_{p}^{(i)}$ vanishes for $i \neq 1$ and is continuous for $i=1$.

We remark that we did not use the full strength of the hypotheses in the proof of the proposition. It would have been sufficient to assume that $h$ and the curves $\mathscr{C}_{i}$ were $C^{n_{1}+n_{2}+1}$ and that $v$ is in $C_{*}^{n_{1}+n_{2}+1}\left(\Omega \backslash \cup \mathscr{C}_{i}\right)$. Secondly, one can easily construct a counterexample which shows that under hypotheses (i), (ii), (iii), the hypothesis (iv) with $k \leqq n_{1}+n_{2}+1$ replaced by $k \leqq n_{1}+n_{2}+2$ is not sufficient to imply the conclusion (v) with $k \leqq n_{1}+n_{2}+1$ replaced by $k \leqq n_{1}+n_{2}+2$. Just let $w^{(i)}, i=1,2$, be two functions which satisfy $\mathrm{w}^{(i)} \in C_{*}^{\infty}\left(\Omega \backslash \mathscr{C}_{i}\right) \cap C^{n_{i}}(\Omega)$ such that some derivative $D^{n_{i}+1} \mathrm{w}^{(i)}$ jumps across $\mathscr{C}_{i}$. Set $v=\mathrm{w}^{(*)}+\mathrm{w}^{(2)}$. Then hypotheses (i)-(iv) hold for all $k$. However, if $h(v)=v^{2}$, then $\left[D^{n_{1}+n_{2}+2} h(v)\right]_{p}^{(i)}$ is not continuous at $p=q$ for $i=1$ or $i=2$.

Next, we show that the jumps of $u$ have higher regularity at $q$ then we expect for $u$ itself. 
Step 4. If $n_{2} \geqq 0$, then for all $|\ell| \leqq n_{1}+n_{2}+2,\left[D^{\ell} u\right]_{p}^{(i)}$ is continuous along each $\mathscr{C}_{i}$ at $p=q$.

Proof. Since $n_{2} \geqq 0, u$ is in $C_{*}^{0}\left(R_{T} \backslash \mathscr{C}_{1}\right)$ so $[u]^{(i)}=0$ on $\mathscr{C}_{i}$ for all $i \neq 1$ and $[u]^{(i)}$ is continuous on $\mathscr{C}_{1}$. We will show that if $\left[D^{\ell} u\right]_{p}^{(i)}$ is continuous for all $i$ and all $|\ell| \leqq k \leqq n_{1}+n_{2}+1$, then the same is true for all $i$ and all $|\ell| \leqq k+1$. Since the hypothesis is valid for $k=0$, the desired result will follow.

Fix $i$. The arguments for the components $u_{i}$ and $u_{j}, j \neq i$, are different. First we consider $u_{i}$ and work in the variables $\left\langle y^{i}, s^{i}\right\rangle$ given by the diffeomorphism $\eta_{i}$. $D^{k+1} u_{i}=\partial_{y}^{\ell_{1}} \partial_{s}^{\ell_{2}} u_{i} \quad$ where $\ell_{1}+\ell_{2}=k+1$. If $\ell_{2} \geqq 1$, then $\left[\partial_{y}^{\ell_{1}} \partial_{s}^{\ell_{2}} u_{i}\right]_{p}^{(i)}=$ $\left[\partial_{y}^{\ell_{1}} \partial_{s}^{\ell_{z}-1} f_{i}(u)\right]_{p}^{(i)}$ is continuous by the induction hypothesis and Proposition 3.1 . Thus, we need only consider $\left[\partial_{y}^{k+1} u_{i}\right]_{p}^{(i)}$. If $\mathscr{C}_{i}$ is given by the line $\left\{\left\langle y_{0}, s\right\rangle\right\}$, then for $y \neq y_{0}(2.4)$ yields

$$
\begin{aligned}
\partial_{y}^{k+1} u_{i}\left(y, s_{0}\right)= & \partial_{y}^{k+1} u_{i}(y, 0)+\int_{0}^{s_{0}} \partial_{y}^{k+1} f_{i}(u(y, r)) d r \\
& -\sum_{j \neq i} \sum_{\ell=0}^{k}\left[\left(\partial_{y}+\gamma_{i}^{\prime} \partial_{s}\right)^{\ell} \gamma_{j}^{\prime} \partial_{y}^{k-\ell} f_{i}(u)\right]_{\left\langle y, \gamma_{j}(y)\right\rangle}^{(j)}
\end{aligned}
$$

By proposition 3.1 and the induction hypothesis the jump terms are continuous at $q$. Therefore, as in the proof of Step 2 (see Fig. 10) we have

$$
\left[\partial_{y}^{k+1} u_{i}\right]_{\left\langle y_{0}, s\right\rangle}^{(i)}=\int_{0}^{s}\left[\partial_{y}^{k+1} f_{i}(u)\right]_{\left\langle y_{0}, r\right\rangle}^{(i)} d r+\left[\partial_{y}^{k+1} u_{i}\right]_{\left\langle y_{0}, 0\right\rangle}^{(i)}
$$

Since the second term on the right is independent of $s$ and the integrand of the first term is bounded, we conclude that $\left[\partial_{y}^{k+1} u_{i}\right]_{\left\langle y_{0}, s\right\rangle}^{(i)}$ is continuous in $s$.

Next, we suppose that $j \neq i$ and work in the variables $\left\langle y^{j}, s^{j}\right\rangle$ given by the diffeomorphism $\eta_{j}$. Let $p=\left\langle y_{0}, s_{0}\right\rangle$ be on $\mathscr{C}_{i}, p_{1}=\left\langle y_{0}, s_{0}+\varepsilon\right\rangle, p_{2}=\left\langle y_{0}, s_{0}-\varepsilon\right\rangle$. As above, if $\ell_{2} \geqq 1$, then $\left[D^{k+1} u_{j}\right]_{p}^{(i)}=\left[\partial_{y}^{\ell_{1}} \partial_{s}^{\ell_{2}} u_{j}\right]_{p}^{(i)}=\left[\partial_{y}^{\ell_{1}} \partial_{s}^{\ell_{2}-1} f_{j}(u)\right]_{p}^{(i)}$ is continuous in $p$ along $\mathscr{C}_{i}$ by Proposition 3.1 and the induction hypothesis. Thus, we need only consider $\left[\partial_{y}^{k+1} u_{j}\right]_{p}^{(i)}$. According to (2.4),

$$
\begin{aligned}
\partial_{y}^{k+1} u_{j}\left(p_{1}\right)= & \partial_{y}^{k+1} u_{j}\left(p_{2}\right)+\int_{s_{0}-\varepsilon}^{s_{0}+\varepsilon} \partial_{y}^{k+1} f_{j}\left(u\left(y_{0}, r\right)\right) d r \\
& -\sum_{\ell=0}^{k}\left[\left(\partial_{y}+\gamma_{i}^{\prime} \partial_{s}^{\ell} \gamma_{i}^{\prime} \partial_{y}^{k-\ell} f_{j}(u)\right]_{\left\langle y_{0}, \gamma_{i}\left(y_{0}\right)\right\rangle}^{(i)} .\right.
\end{aligned}
$$

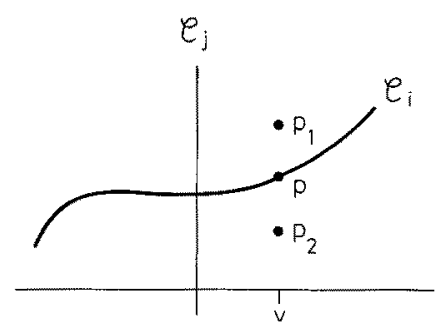


Letting $\varepsilon \downarrow 0$ we find that

$$
\left[\partial_{y}^{k+1} u_{j}\right]_{\left\langle y_{0}, y_{i}\left(y_{0}\right)\right\rangle}^{(i)}=-\sum_{\ell=0}^{k}\left[\left(\partial_{y}+\gamma_{i}^{\prime} \partial_{s}\right)^{\ell} \gamma_{i}^{\prime} \hat{\partial}^{k-\ell} f_{j}(u)\right]_{\left\langle y_{0}, \gamma_{i}\left(y_{0}\right)\right\rangle}^{(i)}
$$

Since $\gamma_{i}$ is $C^{\infty}$, Proposition 3.1 and the induction hypothesis on $u$ imply that each term on the right hand side of (3.6) is continuous in $y_{0}$. Thus, the same is true of the left hand side.

The following theorem describes the local interactions of singularities.

Theorem 2. Let $\mathscr{C}_{i}, i=1, \ldots, m$, be the $m$ characteristic curves of the system (1.2) though a point $q$ (see Fig. 9). Suppose that $u \in L^{\infty}$ is a solution of (1.2) in a neighborhood $R$ of $q$ and that $u \in C_{*}^{n_{1}+n_{2}+2}\left(R \backslash \cup \mathscr{C}_{i}\right)$ where $n_{i}$ is the largest integer (or infinity) such that $u$ is $C^{n_{i}}$ across the incoming part, $\mathscr{C}_{i}^{-}$, of $\mathscr{C}_{i}$ and we label the $n_{i}$ so that $n_{1} \leqq n_{2} \leqq n_{i}$ for $i \neq 1,2$. Then, for each $i, u$ is at least $C^{m_{i}}$ across the outgoing part, $\mathscr{C}_{i}^{+}$, of $i$ where

$$
m_{i}=\min \left\{n_{i}, n_{1}+n_{2}+2\right\} .
$$

Proof. Assume $n_{2} \geqq 0$. For $i=1$ and $i=2$, the conclusion of this theorem is contained in Steps one and two. If $i \neq 1,2$, we already know by Step 2 that $u$ is $C^{n_{2}}$ across the outgoing part of $\mathscr{C}_{i}$ (hereafter denoted by $\mathscr{C}_{i}^{+}$). Let $k$ satisfy $n_{2} \leqq k \leqq n_{1}+n_{2}+1$ and $k \leqq n_{i}-1$ and suppose that $u$ is $k$ times continuously differentiable across $\mathscr{C}_{i}^{+}$with $i \neq 1,2$. We will show that the same statement holds for $k+1$ thus proving the theorem.

Fix $i \neq 1,2$. To look at $u_{j}$ for $j \neq i$ we work in the variables $\left\langle y^{j}, s^{j}\right\rangle$ given by the diffeomorphism $\eta_{j}$. Suppose that $\ell_{1}+\ell_{2}=k+1$ and $\ell_{2} \geqq 1$. Then $\partial_{y}^{\ell_{1}} \partial_{s}^{\ell_{2}} u_{j}=$ $\partial_{y}^{\ell_{1}} \partial_{s}^{\ell_{2}-1} f_{j}(u)$ is smooth across $\mathscr{C}_{i}^{+}$by the induction hypothesis. If $\ell_{2}=0$, then the induction hypothesis and formula (3.6) in Step 4 show that $\left[\partial_{y}^{k+1} u_{j}\right]_{p}^{(i)}=0$, so $u_{j}$ is $C^{k+1}$ across $\mathscr{C}_{i}^{+}$.

For $j=i$, we work in the variables $\left\langle y^{i}, s^{i}\right\rangle$ given by the diffeomorphism $\eta_{i}$. As usual $\partial_{y}^{\ell_{1}} \partial_{s}^{\ell_{2}} u_{i}$ will be continuous across $\mathscr{C}_{i}^{+}$if $\ell_{2} \geqq 1$ so we need only consider $\partial_{y}^{k+1} u_{i}$. We now apply formula (3.5) observing that $\left[\partial_{y}^{k+1} u_{i}\right]_{\left\langle y_{0}, 0\right\rangle}^{(i)}=0$ since $k \leqq$ $n_{i}-1$. Thus,

$$
\left[\partial_{y}^{k+1} u_{i}\right]_{\left\langle y_{0}, s_{0}\right\rangle}^{(i)}=\int_{0}^{s_{0}}\left[\partial_{y}^{k+1} f_{i}(u)\right]_{\left\langle y_{0}, r\right\rangle}^{(i)} d r .
$$

$\left[\partial_{y}^{k+1} f_{i}(u)\right]_{\left\langle y_{0}, r\right\rangle}^{(i)}$ is a sum of terms. Any term which does not contain a $k+1^{s t}$ derivative of $u$ is continuous across $\mathscr{C}_{i}$ by the induction hypothesis and thus its jump is zero. If the $(k+1)^{s t}$ derivative falls on a $u_{j}$ with $j \neq i$, then the jump of that term is zero since we have already proven that $D^{k+1} u_{j}$ is continuous across $\mathscr{C}_{i}$. Only one term, $\left(\partial_{u_{i}} f_{i}\right)\left(\partial_{y}^{k+1} u_{i}\right)$ remains. Since $n_{2} \geqq 0, u$ is continuous across $\mathscr{C}_{i}$ away from $q$ so

$$
\left[\left(\partial_{u_{i}} f_{i}\right)\left(\partial_{y}^{k+1} u_{i}\right)\right]_{\left\langle y_{0}, r\right\rangle}=\left(\partial_{u_{i}} f_{i}\right)\left(u\left(y_{0}, r\right)\right)\left[\partial_{y}^{k+1} u_{i}\right]_{\left\langle y_{0}, r\right\rangle}^{(i)} .
$$


Thus, from (3.7), we obtain,

$$
\left[\partial_{y}^{k+1} u_{i}\right]_{\left\langle y_{0}, s_{0}\right\rangle}^{(i)}=\int_{0}^{s_{0}}\left(\partial_{u_{i}} f_{i}\right)\left(u\left(y_{0}, r\right)\right)\left[\partial_{y}^{k+1} u_{i}\right]_{\left\langle y_{0}, r\right\rangle}^{(i)} d r
$$

which, by Gronwall's inequality, shows that $\left[\partial_{y}^{k+1} u_{i}\right]_{\left\langle y_{0}, s_{0}\right\rangle}^{i\rangle}=0$. This completes the proof that $u$ is $C^{k+1}$ across $\mathscr{C}_{i}^{+}$and concludes the proof of the theorem in the case $n_{2} \geqq 0$.

In the case $n_{1}=-1=n_{2}$, we are only required to prove that $u$ is continuous across $\mathscr{C}_{i}$ for $i \neq 1,2$. This is easily accomplished (without differentiation formulas or the machinery of this section) by using the integral expressions for the $u_{i}$.

In order to apply this local result to the initial value problem in the original region $R_{T}$, we need only introduce some terminology. Let $p$ be a point of $S \cap R_{T}$ which is not in a closed forward cone (defined in the introduction) of $S$. Then, if $\mathscr{N}$ is a small enough neighborhood of $p, \mathscr{N} \cap S$ consists of finitely many pieces of characteristic arcs. If $p$ is an intersection point of two or more of these arcs, then the hypothesis $u \in C_{*}^{n_{1}+n_{2}+2}(\mathscr{N} \backslash S)$ automatically holds since, by Theorem 1 , we know that $u \in C_{*}^{\infty}(\mathscr{N} \backslash S)$. If $p$ is not an intersection point, it lies on a piece of characteristic arc, $\Gamma$, between two intersection points, between an intersection point and the boundary of a closed forward cone, or between an intersection point and the boundary of $R_{r}$. If $\Gamma$ is in $S_{0}$, we define the order of $\Gamma$ to be the order of the singularity of the initial data at the point where $\Gamma$ intersects the line $t=0$. If $\Gamma$ lies in $S_{k}$ for $k \geqq 1$, then $\Gamma$ starts at an intersection point $q$ of characteristic $\operatorname{arcs}$ in $\bigcup_{\ell=0}^{k} S_{\ell}$. Let $n_{i}$ be the orders of the incoming arcs at $q$. Then the order of $\Gamma$ is defined to be $\min \left\{n_{j}+n_{\ell}+2\right\}$. Theorem 2 immediately implies: $j \neq \ell$

Corollary. Let $u \in L^{\infty}\left(R_{T}\right)$ satisfy (1.2) in the sense of distributions. Let $p$ be a point of $S \cap R_{T}$ which is not in a closed forward cone of $S$ and which is not an intersection point of $\operatorname{arcs}$ in $S$. Suppose that the arc on which $p$ lies has order $n$. Then, $u$ is $C^{n}$ at $p$.

\section{Existence of Singularities}

In the last section we showed that when two characteristics bearing singularities of orders $n_{1}$ and $n_{2}$ intersect at a point $q$ (see Fig. 9) then $u$ has order at least $n_{1}+n_{2}+2$ along $\mathscr{C}_{i}^{+}$the outgoing portion of $\mathscr{C}_{i}$, for each $i \neq 1,2$ (assuming that $n_{i}$, the order of $u$ on $\mathscr{C}_{i}^{-}$, is $\geqq n_{1}+n_{2}+2$ ). There is nothing in this statement that prevents $u$ from being $C^{\infty}$ across $\mathscr{C}_{i}^{+}$in which case there would be no anomalous singularity propagating along $\mathscr{C}_{i}^{+}$. In fact, this can happen as one can see by letting time run backward in Fig. 2. In this section we derive a sufficient condition which guarantees that the order of $u$ on $\mathscr{C}_{i}^{+}$is exactly $\min \left\{n_{i}, n_{1}+n_{2}+1\right\}$. Throughout we will use the region, figures, and notation introduced in Sect. 3 . We begin with the case where $n_{i} \leqq n_{1}+n_{2}+1$.

Lemma 4.1. Suppose that $u$ is $C^{\ell}$ across an isolated arc of a characteristic $\mathscr{C}_{i} \subset S$ containing no intersection points of $S$. Then $u_{j}$ is $C^{\ell+1}$ across $\mathscr{C}_{i}$ for all $j \neq i$. 
Proof. We compute in the coordinates $\left\langle y^{j}, s^{j}\right\rangle$ given by the diffeomorphism $n_{j}$. Let $\ell_{1}+\ell_{2}=\ell+1$. Then $\partial_{s}^{\ell_{1}} \partial_{y}^{\ell_{2}} u_{j}=\partial_{y}^{\ell_{1}} \partial_{s}^{\ell_{2}-1} f_{j}(u)$ is continuous across $\mathscr{C}_{i}$ if $\ell_{2} \geqq 1$ by the hypothesis on $u$. And $\partial_{y}^{\ell+1} u_{j}$ is seen to be continuous by using the formula (2.4) and the fact that the jump terms vanish since $u$ is $C^{\ell}$ across $\mathscr{C}_{i}$.

Proposition 4.2. Suppose that, for some $i, u$ has finite order $n_{i} \geqq 0$ on $\mathscr{C}_{i}^{-}$. For each $t \in\left[0, \frac{1}{2}\right)$, let $p_{i}(t)$ be the corresponding point on $\mathscr{C}_{i}^{-}$at time $t$. Then $\left[\partial_{x}^{n_{i}+1} u\right]_{p_{i}(t)}^{(i)} \neq 0$ for all $t \in\left[0, \frac{1}{2}\right)$ and $\lim \left[\partial_{x}^{n_{i}+1} u_{i}\right]_{p_{i}(t)}^{(i)} \neq 0$.

$$
t \uparrow 1 / 2
$$

Proof. We work in the $\left\langle y^{i}, s^{i}\right\rangle$ coordinates in which $\mathscr{C}_{i}$ becomes the line $\left\{\left\langle y_{0}, s\right\rangle\right\}$. For $J \neq i$, Lemma 4.1 shows that $u_{j}$ is $C^{n_{i}+1}$ across $\mathscr{C}_{i}^{-}$. Using the differential equation, we see that the only derivative of $u_{i}$ of order $\leqq n_{i}+1$ which can jump is $\partial_{y}^{n_{i}+1} u_{i}$. By hypothesis this does jump for some $s_{0}<s_{1 / 2}$ where $s_{1 / 2}$ is the value of $s$ corresponding to $t=1 / 2$ in the $\left\langle y^{i}, s^{i}\right\rangle$ coordinate system. From the differential equation, we have

$$
\left[\partial_{y}^{n_{i}+1} u_{i}\right]_{\left\langle y_{0}, s_{0}\right\rangle}^{(i)}=\left[\partial_{y}^{n_{i}+1} u_{i}\right]_{\langle y, s\rangle}^{(i)}+\int_{s}^{s_{0}}\left[\partial_{y}^{n_{i}+1} f_{i}(u)\right]_{\left\langle y_{0}, r\right\rangle}^{(i)} d r
$$

for all $s \in\left[0, s_{1 / 2}\right)$. By the hypotheses on $u$ and Lemma 4.1,

$$
\left[\partial_{y}^{n_{i}+1} f_{i}(u)\right]_{\langle y 0, r\rangle}^{(i)}=\left(\partial_{u_{i}} f_{i}\right)\left[\partial_{y}^{n_{i}+1} u_{i}\right]_{\langle y, r\rangle}^{(i)}
$$

since $n_{i} \geqq 0$ and the jumps of all the other terms vanish. Since $u$ is bounded in $R,\left|\partial_{u_{i}} f_{i}\right| \leqq M$. Thus, by Gronwall's inequality,

$$
\left|\left[\partial_{y}^{n_{i}+1} u_{i}\right]_{\left\langle y_{0}, s_{0}\right\rangle}^{(i)}\right| \leqq e^{M\left|s-s_{0}\right|}\left|\left[\partial_{y}^{n_{i}+1} u_{i}\right]_{\left\langle y_{0}, s\right\rangle}^{(i)}\right| .
$$

Since the left hand side is non-zero, we conclude that $\left[\partial_{y}^{n_{i}+1} u_{i}\right]_{\left\langle y_{0}, s\right\rangle}^{(i)}$ is non-zero for all $s \in\left[0, s_{1 / 2}\right)$ and it cannot approach zero as $s \uparrow s_{1 / 2}$. This proves the result in the coordinate system $\left\langle y^{i}, s^{i}\right\rangle$ and the result in the $\langle x, t\rangle$ coordinates follows.

Proposition 4.3. Let $m_{i}, n_{i}, q$ and the region $R$ be as described in Sect. 3 and Fig. 9. Suppose that for some $i, n_{i} \leqq n_{1}+n_{2}+1$. Then $m_{i}=n_{i}$; i.e. $u$ has order exactly $n_{i}$ across $\mathscr{C}_{i}^{+}$.

Proof. We already know from Theorem 2 that $u$ is $C^{n_{i}}$ across $\mathscr{C}_{i}^{+}$. We must show that $u$ is not $C^{n_{i}+1}$ across $\mathscr{C}_{i}^{+}$. By Proposition 4.2,

$$
\lim _{p \rightarrow q}\left[\partial_{x}^{n_{i}+1} u_{i}\right]_{p}^{(i)}=\alpha \neq 0
$$

But, by hypothesis, $n_{i}+1 \leqq n_{1}+n_{2}+2$, so, by Step 4 of Sect. $3,\left[\partial_{x}^{n_{i}+1} u_{i}\right]_{p}^{(i)}$ is continuous near $q$. Thus $\left[\partial_{x}^{n_{i}+1} u_{i}\right]_{p}^{(i)}$ is non-zero for $p$ on $\mathscr{C}_{i}^{+}$near $q$. The same proof as in Proposition 4.2. shows that $\left[\partial_{x}^{n_{i}+1} u_{i}\right]_{p}^{(i)}$ will be non-zero everywhere on $\mathscr{C}_{i}^{+}$ up to the next intersection point of curves in $S$.

Proposition 4.3 says that an incoming singularity of order $\leqq n_{1}+n_{2}+1$, including those on $\mathscr{C}_{1}$ and $\mathscr{C}_{2}$, will emerge intact from the interaction at $q$. We turn now to the question of the existence of the anomalous singularities produced at $q$. To give as simple a sufficient condition for existence as possible we will assume that for $i \neq 1,2, u$ has order at least $n_{1}+n_{2}+3$ on $\mathscr{C}_{i}^{-}$. This covers the main case 
of interest, i.e. the case when exactly two singularities meet. We also assume that $n_{1} \geqq 0, n_{2} \geqq 0$ which simplifies both the sufficient condition and the proof.

Theorem 3. Let $\mathscr{C}_{i}, i=1,2, \ldots, m$, be the $m$ characteristic curves of the system (1.2) through a point $q$ (see Fig. 9). Suppose that $u \in L^{\infty}$ is a solution of (1.2) in a neighborhood $R$ of $q$ and that $u \in C_{*}^{n_{1}+n_{2}+3}\left(R \backslash \cup \mathscr{C}_{i}\right)$ where $n_{i}, i=1, \ldots, m$ are defined as in Theorem 2 . Suppose that $n_{1} \geqq 0$ and $n_{i} \geqq n_{0}+n_{2}+3$ for $i \neq 1,2$. Then for each $i \neq 1,2, u$ is not $C^{n_{1}+n_{2}+3}$ across $\mathscr{C}_{i}^{+}$near $q$ if and only if

$$
\frac{\partial^{2} f_{i}}{\partial u_{1} \partial u_{2}}(q, u(q)) \neq 0
$$

Proof. Fix $i$, we will work in a variant of the coordinates $\left\langle y^{i}, s^{i}\right\rangle$ given by $\eta_{i}$. Let $\tau^{i}=s^{i}-\gamma_{1}(y)$ where $\gamma_{1}(y)$ is the function whose graph is $\mathscr{C}_{1}$ near $q$. In the coordinates $\langle y, \tau\rangle$ (as usual, we drop the superscripts), all the $i$ th characteristic curves are straight lines of the form $\left\{\left\langle y_{0}, \tau\right\rangle, \tau \geqq 0\right\}$; we take $y_{0}=0$ for $\mathscr{C}_{i}$. In addition $\mathscr{C}_{1}$ is the straight line $\tau=0$. See Fig. 12. For $j \neq i$, let $\gamma_{j}(\cdot)$ be the function so that $\mathscr{C}_{j}$ is the graph $\left\{\left\langle y, \gamma_{j}(y)\right\rangle\right\}$ in the new coordinates. Using (2.4), we find for $y$ close to zero, and $\tau>0$,

$$
\begin{aligned}
\partial_{y}^{n_{1}+n_{2}+3} u_{i}(y, \tau)= & \partial_{y}^{n_{1}+n_{2}+3} u_{i}\left(y, \tau_{0}\right)+\int_{\tau_{0}}^{\tau} \partial_{y}^{n_{1}+n_{2}+3} f_{i}(u(y, r)) d r \\
& -\sum_{\ell=0}^{n_{1}+n_{2}+2}\left[\left(\partial_{y}+\gamma_{2}^{\prime} \partial_{s}\right)^{\ell} \gamma_{2}^{\prime} \partial_{y}^{n_{1}+n_{2}+2-\ell} f_{i}(u)\right]_{\left\langle y, \gamma_{2}(y)\right\rangle}^{(2)}
\end{aligned}
$$

where $\tau_{0}<0$ is small and fixed. There are no jump terms across $\mathscr{C}_{j}$ for $j \neq 1,2$ since we know by Theorem 2 that $u$ is at least $n_{1}+n_{2}+2$ across those curves. There are no jumps for $j=1$ because in our coordinates $\gamma_{1}^{\prime}(y) \equiv 0$. Since $u$ has order at least $n_{1}+n_{2}+3$ on $\mathscr{C}_{i}^{-}, \partial_{y}^{n_{1}+n_{2}+3} u_{i}\left(y, \tau_{0}\right)$ is continuous at $y=0$. Thus, setting

$$
J(y) \equiv \sum_{\ell=0}^{n_{1}+n_{2}+2}\left[\left(\partial_{y}+\gamma_{2}^{\prime} \partial_{s}\right)^{\prime} \gamma_{2}^{\prime} \partial_{y}^{n_{1}+n_{2}+2-\ell} f_{i}(u)\right]_{\left\langle y, \gamma_{2}(y)\right\rangle}^{(2)}
$$

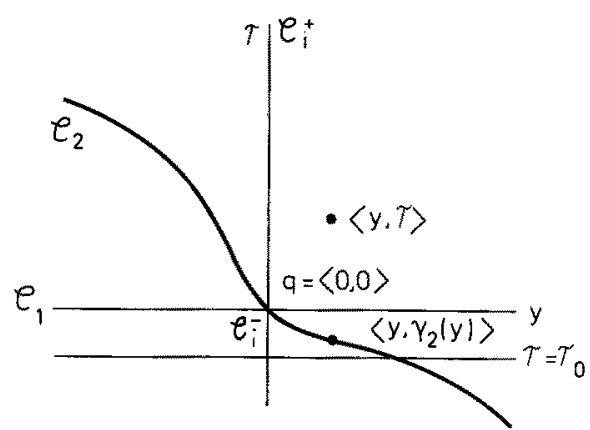

Fig. 12 
we have

$$
\left[\partial_{y}^{n_{1}+n_{2}+3} u_{i}\right]_{\langle 0, \tau\rangle}^{(i)}=\int_{\tau_{0}}^{\tau}\left[\partial_{y}^{n_{1}+n_{2}+3} f_{i}(u)\right]_{\langle 0, r\rangle}^{(i)} d r+L
$$

where

$$
L=\lim _{\varepsilon \downarrow 0} J(\varepsilon)-J(-\varepsilon) .
$$

Since $u$ is $C^{n_{1}+n_{2}+2}$ across $\mathscr{C}_{i}$, Lemma 4.1 implies that there is only one non-zero terms in the integrand, namely $\left(\partial_{u_{i}} f_{i}\right)\left[\partial_{y}^{n_{1}+n_{2}+3} u_{i}\right]^{(i)}$. This term vanishes for $r<0$ since $u$ has order at least $n_{1}+n_{2}+3$ on $\mathscr{C}_{i}^{-}$. Thus, we have

$$
\left[\partial_{y}^{n_{1}+n_{2}+3} u_{i}\right]_{\langle 0, \tau\rangle}^{(i)}=\int_{0}^{\tau}\left(\partial_{u_{i}} f_{i}(u)\right)\left[\partial_{y}^{n_{1}+n_{2}+3} u_{i}\right]_{\langle 0, r\rangle}^{(i)} d r+L .
$$

Since $\partial_{y}^{n_{1}+n_{2}+3} u_{i}$ is bounded, $\left[\partial_{y}^{n_{1}+n_{2}+3} u_{i}\right]_{\langle 0, \tau\rangle}^{(i)}$ will be non-zero for $\tau$ small if $L \neq 0$. Conversely, if $L=0$, then Gronwall's inequality shows that $\left[\partial_{y}^{n_{1}+n_{2}+3} u_{i}\right]_{\langle 0, \tau\rangle}^{(i)}=0$. Thus $u$ will be $C^{n_{1}+n_{2}+3}$ across $\mathscr{C}_{i}^{+}$if and only if $L=0$.

We shall compute $L$ and show that $L \neq 0$ if and only if (4.1) holds. We repeatedly use the following simple lemma. As usual $[h]_{p}^{(i)}$ will denote the jump of $h$ from below $\mathscr{C}_{i}$ to above $\mathscr{C}_{i}$ at $p$.

Lemma 4.4. Suppose that $\mathscr{C}_{1}, \mathscr{C}_{2}$, the graphs of two smooth functions $\gamma_{1}, \gamma_{2}$, intersect transversally at $q=\langle 0,0\rangle$. Let $\Omega$ be a neighborhood of $q$ and let $v$ and $w$ be functions on $\Omega \backslash\left(\mathscr{C}_{1} \cup \mathscr{C}_{2}\right)$ which satisfy

(i) $v \in C_{*}^{0}\left(\Omega \backslash \mathscr{C}_{1}\right)$

(ii) $w \in C_{*}^{0}\left(\Omega \backslash\left(\mathscr{C}_{1} \cup \mathscr{C}_{2}\right)\right)$ and $[w]_{p}^{(2)}$ is continuous for $p$ near $q$.

Define

$$
\phi(y)=[v w]_{\left\langle y, \gamma_{z}(y)\right\rangle}^{(2)} .
$$

Then

$$
\lim _{y \downarrow 0} \phi(y)-\phi(-y)= \pm[v]_{q}^{(1)}[w]_{q}^{(2)} .
$$

Proof. There are two cases to consider depending on the relationship of $\mathscr{C}_{1}$ and $\mathscr{C}_{2}$ (see Fig. 13). Suppose that we are in the case depicted in Figure $13 \mathrm{a}$. Then,

$$
\begin{aligned}
& \lim \left\{[v w]_{\left\langle y, \gamma_{2}(y)\right\rangle}^{(2)}-[v w]_{\left\langle-y, \gamma_{2}(-y)\right\rangle}^{(2)}\right\} \\
& \quad=\lim _{y \downarrow 0}\left\{v\left(y, \gamma_{2}(y)\right)[w]_{\left\langle y, \gamma_{2}(y)\right\rangle}^{(2)}-v\left(-y, \gamma_{2}(-y)\right)[w]_{\left\langle-y, \gamma_{2}(-y)\right\rangle}^{(2)}\right\} \\
& \quad=[v]_{q}^{(1)}[w]_{q}^{(2)} .
\end{aligned}
$$

a)

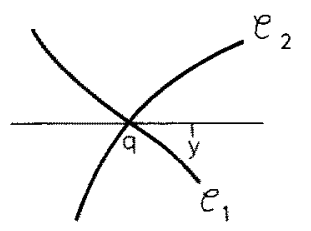

b)

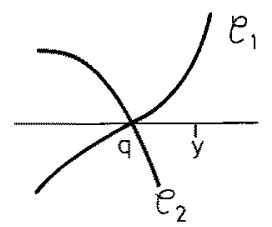

Fig. 13a and $b$ 
The first step used the hypothesis that $v$ is continuous across $\mathscr{C}_{2}$ and the second step used the hypothesis that $[w]_{p}^{(2)}$ is continuous for $p$ near $q$. In the case depicted in Fig. $13 \mathrm{~b}$ the calculation and result are the same except for a minus sign.

We now return to the proof of Theorem 3. Whenever we use Lemma 4.4 we will also be using the result of Step 4 of Sect. 3 which guarantees that all the jumps $\left[D^{\ell} u\right]_{p}^{(i)}$ are continuous for $p$ near $q$ if $|\ell| \leqq n_{1}+n_{2}+2$. We set $k=n_{1}+n_{2}+2$. In order to compute $L$, we deal with each of the terms

$$
T_{\ell}(y)=\left[\left(\partial_{y}+\gamma_{2} \partial_{s}\right)^{\ell} \gamma_{2}^{\prime} \partial_{y}^{k-\ell} f_{i}(u)\right]_{\left\langle y, \gamma_{2}(y)\right\rangle}^{(2)}
$$

separately. Using Leibnitz' rule we can write $T_{\ell}$ as a sum of terms of the form

$$
\begin{aligned}
j_{\alpha, \delta, \beta}(y) & =\left[\left(D^{\alpha} f_{i}(u)\right)\left(\partial_{y}^{\delta} \gamma_{2}^{\prime}\right) \prod_{j \geqq 1} D^{\beta_{j}} u_{j}\right]_{\left\langle y, \gamma_{2}(y)\right\rangle}^{(2)} \\
& =\left(D ^ { \alpha } f _ { i } ( u ( y , \gamma _ { 2 } ( y ) ) ) \left(\partial_{y}^{\left.\delta_{\gamma} \gamma_{2}^{\prime}\right)}\left[\prod_{j \geqq 1} D^{\beta_{j}} u_{n(j)}\right]_{\left\langle y, \gamma_{2}(y)\right\rangle}^{(2)}\right.\right.
\end{aligned}
$$

where $\sum\left|\beta_{j}\right| \leqq n_{1}+n_{2}+2$ and each $D$ stands for

$$
\left(\partial_{y}+\gamma_{2}^{\prime} \partial_{s}\right) \text { or } \partial_{y} \text {. }
$$

$D^{\alpha} f_{i}(u)$ and $\partial_{y}^{\delta} \gamma_{2}^{\prime}$ come out of the jump bracket because they are both continuous since $n_{j} \geqq 0$ for all $j$ and $\gamma_{2}$ is smooth. We next investigate when the limit

$$
L_{\alpha, \delta}^{\beta} \equiv \lim _{y \downarrow 0} j_{\alpha, \delta, \beta}(y)-j_{\alpha, \delta, \beta}(-y)
$$

can be non-zero. According to Lemma 4.4, $\prod_{j=1}^{m} D^{\beta_{j}} u_{j}$ must have a term which jumps across $\mathscr{C}_{1}$ and another which jumps across $\mathscr{C}_{2}$. If one $\beta_{i}$ satisfies $\left|\beta_{i}\right|>n_{2}+1$ then the rest satisfy $\left|\beta_{j}\right| \leqq n_{1}$. Thus $\prod_{j \neq i} D^{\beta_{j}} u_{n(j)}$ will not jump across $\mathscr{C}_{1}$ or $\mathscr{C}_{2}$ so $L_{\alpha, \delta}^{\beta}=0$. On the other hand, if all $\beta_{j}$ satisfy $\left|\beta_{j}\right| \leqq n_{2}$ then none of the factors jump across $\mathscr{C}_{2}$ so again $L_{\alpha, \delta}^{\beta}=0$. Thus one $\beta_{j}$ must equal $n_{2}+1$, call it $\beta_{1}$. As above, if $\left|\beta_{j}\right| \leqq n_{1}$ for all $j>1$, Lemma 4.4 implies that $L_{\alpha, \delta}^{\beta}=0$. Since $\sum_{j>1}\left|\beta_{j}\right| \leqq n_{1}+1$, we conclude that $L_{\alpha, \delta}^{\beta}$ will be zero unless there is exactly one non-zero $\beta_{j}$ for $j>1$, call it $\beta_{2}$, and it must satisfy $\left|\beta_{2}\right|=n_{1}+1$. Thus $L_{\alpha, \delta}^{\beta}=0$ except possibly for terms of the form, $|\alpha|=2$,

$$
\gamma_{2}^{\prime}\left(D^{\alpha} f_{i}(u)\right)\left[\left(D^{n_{1}+1} u_{j(1)}\right)\left(D^{n_{2}+1} u_{j(2)}\right)\right]_{\left\langle y, y_{2}(y)\right\rangle}^{(2)} .
$$

Suppose $n_{1}<n_{2}$. Then, we must have $j(2)=2$ since if $j(2) \neq 2$, Lemma 4 .1 shows that $D^{n_{2}+1} u_{j(2)}$ will not jump across $\mathscr{C}_{2}$ in which case $L_{\alpha, \beta}^{\beta}=0$ by Lemma 4.4 . The same argument shows that we must have $j(1)=1$ and a similar argument handles the case $n_{1}=n_{2}$. Therefore, to compute $L_{\ell} \equiv \lim T_{\ell}(y)-T_{\ell}(-y)$ we need only consider terms of the form:

$$
\gamma_{2}^{\prime}(y) \frac{\partial^{2} f_{i}}{\partial u_{1} \partial u_{2}}\left(y, \gamma_{2}(y)\right)\left[\left(D^{n_{1}+1} u_{1}\right)\left(D^{n_{2}+1} u_{2}\right)\right]_{\left\langle y, \gamma_{2}(y)\right\rangle}^{(2)}
$$

where exactly $\ell$ of the $D$ 's equal $\left(\partial_{y}+\gamma_{2}^{\prime} \partial_{s}\right)$ and the other $(k-\ell)$ equal $\partial_{y}$. If any of 
the factors $\left(\partial_{y}+\gamma_{2}^{\prime} \partial_{s}\right)$ is contained in $D^{n_{2}+1}$, then $D^{n_{2}+1} u_{2}=D^{n_{2}} f_{2}(u)$ doesn't jump across $\mathscr{C}_{2}$ so by Lemma $4.4, L_{\alpha, \delta}^{\beta}=0$. We are thus reduced to studying terms of the form

$$
j_{\ell}(y) \equiv\left[\left(\left(\partial_{y}+\gamma_{2}^{\prime} \partial_{s}\right)^{\ell} \partial_{y}^{n_{1}+1-\ell} u_{1}\right)\left(\partial_{y}^{n_{2}+1} u_{2}\right)\right]_{\left\langle y, \gamma_{2}(y)\right\rangle}^{(2)} .
$$

We now write

$$
\left(\partial_{y}+\gamma_{2}^{\prime} \partial_{s}\right)^{\ell} \partial_{y}^{n_{1}+1-\ell}=\left(\left(\partial_{y}+\bar{\gamma}_{1}^{\prime} \partial_{s}\right)+\left(\gamma_{2}^{\prime}-\bar{\gamma}_{1}^{\prime}\right) \partial_{s}\right)^{\ell}\left(\left(\partial_{y}+\bar{\gamma}_{1}^{\prime} \partial_{s}\right)-\bar{\gamma}_{1}^{\prime} \partial_{s}\right)^{n_{1}+1-\ell}
$$

where $\bar{\gamma}_{1}^{\prime}(y)$ is the slope of the first characteristic at $\left\langle y, \gamma_{2}(y)\right\rangle$. If we expand (4.4) and apply the result to $u_{1}$, the differential equation and Lemma 4.4 imply that no term with a factor of $\left(\partial_{y}+\bar{\gamma}_{1}^{\prime} \partial_{s}\right)$ can contribute to $\lim j_{t}(y)-j_{t}(-y)$ since it will not jump across $\mathscr{C}_{1}$. Similarly, we write

$$
\partial_{y}^{n_{2}+1} u_{2}=\left(\left(\partial_{y}+\gamma_{2}^{\prime} \partial_{s}\right)-\gamma_{2}^{\prime} \partial_{s}\right)^{n_{2}+1} u_{2}
$$

and observe that no term with a factor of $\left(\partial_{y}+\gamma_{2}^{\prime} \partial_{s}\right)$ can contribute to the limit. We are thus reduced to studying

$$
\phi_{\ell}(y) \equiv\left(\gamma_{2}^{\prime}-\bar{\gamma}_{1}^{\prime}\right)^{\ell}\left(-\bar{\gamma}_{1}^{\prime}\right)^{n_{1}+1-\ell}\left(-\gamma_{2}^{\prime}\right)^{n_{2}+1}\left[\left(\partial_{s}^{n_{1}+1} u_{1}\right)\left(\partial_{s}^{n_{2}+1} u_{2}\right)\right]_{\left\langle y, \gamma_{2}(y)\right\rangle}^{(2)} .
$$

Notice that $\bar{\gamma}_{1}^{\prime}(y) \rightarrow \bar{\gamma}_{1}^{\prime}(0)=\gamma_{1}^{\prime}(0)=0$ as $y \rightarrow 0$ since $\langle 0,0\rangle$ is on both $\mathscr{C}_{1}$ and $\mathscr{C}_{2}$. Therefore, $\lim _{y \downarrow 0} \phi_{\ell}(y)-\phi_{\ell}(-y)=0$ unless $\ell=n_{1}+1$. If $n_{1}<n_{2}, \partial_{s}^{n_{1}+1} u_{1}$ is automatically continuous across $\mathscr{C}_{2}$ and if $n_{1}=n_{2}$, then $\partial_{s}^{n_{1}+1} u_{1}$ is continuous across $\mathscr{C}_{2}$ by Lemma 4.1 . This fact and Step 4 of Sect. 3 show that $\partial_{s}^{n_{1}+1} u_{1}$ and $\partial_{s}^{n_{2}+1} u_{2}$ satisfy the hypothesis of Lemma 4.4 , so for $\ell=n_{1}+1$, we obtain

$$
\lim _{y \Downarrow 0} \phi_{n_{1}+1}(y)-\phi_{n_{1}+1}(-y)= \pm \gamma_{2}^{\prime}(0)^{n_{1}+1}\left(-\gamma_{2}^{\prime}(0)\right)^{n_{2}+1}\left[\partial_{s}^{n_{1}+1} u_{1}\right]_{q}^{(1)}\left[\partial_{s}^{n_{2}+1} u_{2}\right]_{q}^{(2)}
$$

Thus, we conclude that

$$
L= \pm \gamma_{2}^{\prime}(0)^{n_{1}+n_{2}+2}\left(\frac{\partial^{2} f_{i}}{\partial u_{1} \partial u_{2}}(q, u(q))\right)\left[\partial_{s}^{n_{1}+1} u_{1}\right]_{q}^{(1)}\left[\partial_{s}^{n_{2}+1} u_{2}\right]_{q}^{(2)}
$$

By strict hyperbolicity, $\mathscr{C}_{2}$ is not tangent to $\mathscr{C}_{1}$ at $q$, so $\gamma_{2}^{\prime}(0) \neq 0$. By Proposition 4.2 , both $\left[\partial_{s}^{n_{1}+1} u_{1}\right]_{q}^{(1)}$ and $\left[\partial_{s}^{n_{2}+1} u_{2}\right]_{q}^{(2)}$ are non-zero. Thus, $L \neq 0$ if and only if $\frac{\partial^{2} f_{i}}{\partial u_{1} \partial u_{2}}(q, u(q))$ is non-zero.

The purpose of Theorem 3 is to show that anomalous singularities of order $n_{1}+n_{2}+2$ are "usually" produced if singularities of order $n_{1}$ and $n_{2}$ intersect. Of course, verification of the condition (4.1) is non-trivial since one normally does not know the solution $u$ explicitly. However, in some cases, for example if $\frac{\partial^{2} f_{i}}{\partial u_{j} \partial u_{k}}$ never vanishes for $i, j, k$ distinct, the condition is automatically satisfied at each intersection point; we give such an example below. On the opposite extreme, suppose that $\frac{\partial^{2} f_{i}}{\partial u_{j} \partial u_{k}} \equiv 0$ for all $i, j, k$ distinct. Then

$$
f_{i}(x, t, u)=\sum_{j \neq i}^{m} h_{j}\left(t, x, u_{j}, u_{i}\right)
$$


for each $i$. In particular, this holds if $F$ is linear. In this case one can show by using the methods of Section 2 that no anomalous singularities of any orders are produced at any intersection points. Roughly speaking, in order to produce an anomalous singularity going in the $i$ th direction from $q$, we need $f_{i}$ to contain products of other components of $u$ which are singular at $q$; see the example below.

We used the hypothesis $n_{1} \geqq 0$ repeatedly in the proof of Theorem 3 because it guaranteed that $u$ and therefore derivatives of the $f_{i}$ evaluated at $\langle x, t, u(x, t)\rangle$ are continuous. In the special case $n_{1}=-1$, the condition analogous to (4.1) is more complicated.

We can now explain how a singularity can disappear. Suppose that we have singularities along $\mathscr{C}_{1}$ and $\mathscr{C}_{2}$ as in Theorem 3 and suppose that there is an incoming singularity on $\mathscr{C}_{i}$ of order exactly $n_{1}+n_{2}+2$. In this case (4.2) will have the additional term $I \equiv\left[\partial_{y}^{n_{1}+n_{2}+3} u_{i}\right]_{\left\langle 0, \tau_{0}\right\rangle}^{(i)}$ on the right since the initial data $\tau=\tau_{0}$ jumps in the $\left(n_{1}+n_{2}+3\right)^{r d}$ derivative. If it just happens that $L+I=0$ then there will be no singularity of order $n_{1}+n_{2}+2$ along $\mathscr{C}_{i}^{+}$. This is what happens when the situation in Fig. 2 is run backwards in time. In that case the cancellation is not surprising since the size of the jump along the dashed line corresponds exactly to the limit of jump terms, $L$, at $\langle 0,1\rangle$.

Suppose that all the hypotheses of Theorem 3 hold except (4.1). Then the theorem tells us that $D^{n_{1}+n_{2}+3} u$ will be continuous across $\mathscr{C}_{i}^{+}$. What about higher derivatives? They will "usually" jump across $\mathscr{C}_{i}^{+}$but the conditions on $f_{i}$ which guarantee the existence of such higher order jumps, when the lowest order condition, (4.1), fails will be more complicated.

Example. Consider the system

$$
\begin{array}{rlrl}
{\left[\frac{\partial}{\partial t}+\frac{\partial}{\partial x}\right] v} & =f_{1} & v(x, 0) & =v_{0}(x) \\
{\left[\frac{\partial}{\partial t}-\frac{\partial}{\partial x}\right] w} & =f_{2} & w(x, 0) & =w_{0}(x) \\
\frac{\partial z}{\partial t} & =f_{3} & z(x, 0) & =z_{0}(x)
\end{array}
$$

and suppose that $v_{0}, w_{0}, z_{0}$ have singularities of orders $n_{1}$ at $x_{1}$ and $n_{2}$ at $x_{2}\left(n_{1} \geqq 0\right)$ but are $C^{\infty}$ elsewhere. If $f_{1}=w z, f_{2}=z v, f_{3}=v w$, then at each intersection point of $S$ (see Figure 1.3) an anomalous singularity will be produced since

$$
\frac{\partial^{2} f_{1}}{\partial w \partial z}=\frac{\partial^{2} f_{2}}{\partial z \partial v}=\frac{\partial^{2} f_{3}}{\partial v \partial w}=1 \neq 0
$$

Thus the entire latticework $S$ in Fig. 3 will be in the singular support of the solution $u=\langle v, w, z\rangle$ and, using Theorem 3 we can say exactly which derivative of $u$ will jump across each line in the lattice. For example, on $S_{1}$ the $\left(n_{1}+n_{2}+3\right)^{r d}$ derivative will jump, on $S_{2}^{\prime \prime}$ the $\left(\left(n_{1}+n_{2}+2\right)+n_{2}+3\right)^{r d}$ derivative will jump, and on $S_{2}^{\prime}$ the $\left(\left(n_{1}+n_{2}+2\right)+n_{1}+3\right)^{\text {rd }}$ derivative will jump.

Actually, the above statements are only valid up to the time when the solution 
blows up (which it will do in finite time). To make a similar example which is global in time, let $f_{1}=e^{-w z}, f_{2}=e^{-v z}, f_{3}=e^{-v w}$, and take the initial data to be $\geqq 2$. Then $v, w, z$ remain $\geqq 2$ since the right hand sides are positive. Since the $f_{i}$ remain bounded the solution will be global in time. Now,

$$
\frac{\partial^{2} f_{1}}{\partial w \partial z}=(z w-1) e^{-w z}
$$

so $\frac{\partial^{2} f_{1}}{\partial w \partial z} \neq 0$ at any intersection point since $z \geqq 2, w \geqq 2$. The same holds for $\frac{\partial^{2} f_{2}}{\partial v \partial z}$ and $\frac{\partial^{2} f_{3}}{\partial v \partial w}$ so condition (4.1) is satisfied at each intersection point of the lattice $S$. Thus all possible anomalous singularities will be produced and the entire lattice $S$ will be the singular support of $u$.

\section{References}

1. Bony, J.-M. : Calcul symbolique et propagation des des singularities pour les équations aux dérivées partielles non linéaires. Preprint (1980)

2. Lascar, B. : C. R. Acad. Sci. (Paris) 287A, 521-529

3. Rauch, J. : J. Math. Pures Appl. 58, 299-308 (1979)

4. Rauch, J., Reed, M. : Ann. Math. 111, 531-552 (1980)

5. Rauch, J., Reed, M. : Nonlinear microlocal analysis of semilinear hyperbolic systems in one space dimension. Preprint, 1981

Communicated by J. Glimm

Received November 10, 1980 